\title{
$S U(3)_{F}$ breaking through final state interactions and $C P$ asymmetries in $D \rightarrow P P$ decays
}

\author{
Franco Buccella* \\ INFN, Sezione di Napoli, via Cintia, 80126 Napoli, Italy \\ Ayan Paul ${ }^{\dagger}$ \\ DESY, Notkestrasse 85, D-22607 Hamburg, Germany \\ and Institut für Physik, Humboldt-Universität zu Berlin, D-12489 Berlin, Germany \\ Pietro Santorelli \\ Dipartimento di Fisica "Ettore Pancini”, Università di Napoli Federico II, \\ Complesso Universitario di Monte S. Angelo, Via Cintia Edificio 6, 80126 Napoli, Italy \\ and INFN, Sezione di Napoli, via Cintia, 80126 Napoli, Italy
}

(Received 14 February 2019; published 11 June 2019)

\begin{abstract}
We analyze $D$ decays to two pseudoscalars $(\pi, K)$ assuming the dominant source of $\mathrm{SU}(3)_{F}$ breaking lies in final state interactions. We obtain an excellent agreement with experimental data and are able to predict $C P$ violation in several channels based on current data on branching ratios and $\Delta \mathrm{A}_{C P}$. We also make predictions for $\delta_{K \pi}$ and the branching fraction for the decay $D_{s}^{+} \rightarrow K^{+} K_{L}$.
\end{abstract}

DOI: $10.1103 /$ PhysRevD.99.113001

\section{INTRODUCTION}

The search for physics beyond the Standard Model (SM) has brought about a search for $C P$ violation beyond what is generated by the phase in the CKM matrix [1-3]. The challenging measurement and conclusive evidence of a nonvanishing value for the ratio $\epsilon^{\prime} / \epsilon$ [4-6] has excluded the superweak hypothesis of Wolfenstein [7]. However, $\epsilon^{\prime} / \epsilon$ has not yet facilitated a severe test of the SM. This is primarily due to the poor predictive power plaguing this ratio in the presence of cancellations between QCD and electroweak penguins. The possibility of a quantitative evaluation of these matrix elements often transforms into a theological debate, in spite of the encouraging and pioneering lattice results by the RBC and UKQCD collaborations [8]. On the other hand, the triumphant measurement of $O$ (1) $C P$ violation in the golden decay channel $J / \psi K_{S}$ [9-12] of the neutral $B$ meson, where the measured timedependent asymmetry depends, to an excellent approximation, only on the $C P$ violating phase of the CKM matrix [13-15], has been a striking confirmation of the SM. More

\footnotetext{
*franco.buccella@na.infn.it †ayan.paul@desy.de "pietro.santorelli@na.infn.it
}

Published by the American Physical Society under the terms of the Creative Commons Attribution 4.0 International license. Further distribution of this work must maintain attribution to the author(s) and the published article's title, journal citation, and DOI. Funded by SCOAP ${ }^{3}$. recently, $\mathrm{LHCb}$ has also confirmed the validity of the SM through a measurement of $C P$ violation in $B_{s}$ physics [16]. $\mathrm{LHCb}$ has also played a key role in bringing the pioneering first results on neutral $D^{0}$ meson mixing by previous experiments [17-42] to a mature stage, along with impressive progress in the measurement of $C P$ violation [43-50].

In the recent past, the controversial measurements [41-45,51-54] of the $C P$ violating asymmetry found in the decay of the neutral $D^{0}$ meson to pairs of charged kaons and pions had effectively stirred the question of whether such rather high values found in the first experimental results could be accommodated within the Standard Model. While many arguments were placed in favor of contributions coming from beyond the SM [55-68], concrete arguments were also made for the presence of large phases coming from final state interactions (FSI) allowing for the accommodation of the asymmetry within the SM [69-76]. In fact, both the isospin relations for the Cabibbo allowed (CA) decays into $\bar{K} \pi$ and singly Cabibbo suppressed (SCS) decays into $\pi \pi$ of $D$ mesons are characterized by large angles in the complex plane for the corresponding triangles $[77,78]$. In the case of the $\pi \pi$ final states the phase difference between the $I=2$ and $I=0$ amplitudes is about $\pi / 2$. These large phases have been, for a long time, advocated as the main cause for the large $S U(3)_{F}$ violations in exclusive $D$ decays $[79,80]$. Indeed, in the $D$ mass region there is a nonet of scalar resonances and their mass splittings imply large $\mathrm{SU}(3)_{F}$ violations generated by FSI.

Identifying the dominant source of $\mathrm{SU}(3)_{F}$ violation is of crucial phenomenological importance in $D$ decays, since 
on one hand the imposition of exact $\mathrm{SU}(3)_{F}$ completely fails in reproducing experimental data, while on the other hand introducing $\mathrm{SU}(3)_{F}$ breaking in a general manner leads to a complete loss of predictivity due to the proliferation of independent parameters (see e.g., Ref. [76]). Several interesting attempts at reducing the number of parameters have been made. The authors of Refs. [81-83] advocated the use of $1 / N_{c}$ counting to reduce the size of the parameter set to a tractable number. However, relying on the $1 / N_{c}^{2}$ suppression of formally divergent corrections seems questionable. In Ref. [70], the dominance of lower rank representations was argued for, and only $\mathrm{SU}(3)_{F}$ triplets were considered as additional operators in the effective Hamiltonian. However, there is no compelling reason to truncate the effective Hamiltonian in such a drastic manner.

In contrast to the above-mentioned approaches, the assumption that FSI is the dominant source of $\mathrm{SU}(3)_{F}$ breaking rests solidly on the large observed strong phases: it provides a very good description of available experimental data, it allows one to predict several $C P$ asymmetries which are currently poorly measured, and it can be tested against independent determinations of the relevant rescattering matrices. It also allows us to predict the relative strong phase between the doubly Cabibbo suppressed (DCS) and CA charged $K \pi$ decays, $\delta_{K \pi}$.

The paper is organized as follows. In the next section we write the amplitudes for all the decays considered. In Sec. III we discuss the parametrization of the $\Delta U=0$ part of the amplitudes proportional to $V_{c b}^{*} V_{u b}$. In Sec. IV we give a brief overview of the current status of experimental measurements of $C P$ asymmetries in SCS decays. Using the experimental branching ratios of $D^{0}, D^{+}$, and $D_{s}^{+}$into final states with kaons and/or pions and measurements of $C P$ asymmetries we fit the values of the parameters in Sec. V. In this section we also take a critical look at our parametrization and its consequences on correlations between $C P$ asymmetries followed by an estimate of how future measurements will improve the constraints on penguin amplitudes. We finally summarize our results in Sec. VI. The details of the fit results are given in the Appendix.

\section{AMPLITUDES AND PARAMETRIZATION}

It is customary to describe weak decay amplitudes in terms of topologies of Wick contractions (or renormalization-group-invariant combinations thereof). Notice that any Wick contraction, as defined in Refs. [84,85], can be seen as an emission followed by rescattering $[84,86,87]$. Thus, rescattering establishes a link between emissions and long-distance contributions to other subleading topologies such as penguins, annihilations, weak exchange, etc. The large phases observed in two-body nonleptonic $D$ decays imply the importance of FSI, leading to an effective description of decay amplitudes in terms of emissions followed by rescattering. This description was developed in Refs. [79,80], where FSI effects were parametrized in terms of resonances [88].

In a previous study [91] of the SCS decays of the $D^{0}$ into a pair of pseudoscalars, exact $\mathrm{SU}(3)_{F}$ symmetry was assumed amongst the emission matrix elements of the nonleptonic Hamiltonian. The necessary $\mathrm{SU}(3)_{F}$ breaking was determined by FSI, described as the effect of resonances in the scattering of the final particles. Assuming no exotic resonances belonging to the 27 representation, the possible resonances have $\mathrm{SU}(3)_{F}$ and isospin quantum numbers $(8, I=1),(8, I=0)$ and $(1, I=0)$. Moreover, the two states with $I=0$ can mix, yielding two resonances:

$$
\begin{aligned}
& \left|f_{0}\right\rangle=\sin \phi|8, I=0\rangle+\cos \phi|1, I=0\rangle, \\
& \left|f_{0}^{\prime}\right\rangle=-\cos \phi|8, I=0\rangle+\sin \phi|1, I=0\rangle .
\end{aligned}
$$

The main contribution from the Hamiltonian, $H(|\Delta C|=1$, $\Delta S=0)$ transforms as a $U$-spin triplet and therefore relates the $D^{0}$, which is a $U$-spin singlet, to the $U$-spin triplets of the 8 and 27 representation of SU(3) $)_{F}$. So in Ref. [91] two parameters were introduced for the matrix elements of the weak Hamiltonian, namely $T$ and $C$. The phase of the $I=1$ octet amplitude, $\delta_{1}$, and the two phases and mixing angle between the $I=0$ singlet and octet amplitude, $\delta_{0}, \delta_{0}^{\prime}$ and $\phi$, were taken as free parameters. The strong phases should be related to the mass and width of the resonances. However, the lack of complete experimental information on the scalar resonances do not allow for the determination of the strong phases and so we determine them from the fit. It should also be noted that notwithstanding the lack of exotic resonances belonging to the 27 representation, there is a small phase associated with this amplitude which is compatible with 0 [78]. We set this phase to 0 and hence all the other phases should be interpreted as a difference with respect to this phase.

Other attempts have been made previously to study $D \rightarrow P P$ decays in the $\mathrm{SU}(3)_{F}$ framework with perturbative breaking of the symmetry $[73,76,81-83,92]$. The spotlight has always been on prescriptions for estimating the penguin amplitudes by formulating a reasonable parametrization in the $\mathrm{SU}(3)_{F}$ framework and then using available data on branching fractions and $C P$ asymmetries. In this work we extend the formalism that was developed in Ref. [91] by including more decay modes of the $D$ meson system and introducing new parameters to aptly parametrize the additional decay amplitudes.

The $D^{+}$and $D_{s}^{+}$form a $U$-spin doublet and the matrix elements of the weak Hamiltonian, which relate $D^{0}$ to the $Q=0, U=1$ and $D^{+}$to the $Q=1, U=\frac{1}{2}$ of the octet, are independent. This requires the introduction of a $\mathrm{SU}(3)_{F}$ invariant parameter $\Delta$ in the $\Delta U=1$ part of the amplitude. The terms proportional to $\Delta$ vanish in the factorization ansatz and appears only in the $D_{(s)}^{+}$decay amplitudes. As we will explain in Sec. III, $\Delta$ is related by $\mathrm{SU}(3)_{F}$ to a 
vanishingly small contribution in the $\Delta U=0$ part of the amplitude suppressed by an approximate selection rule.

To expand the FSI description we need a phase, $\delta_{\frac{1}{2}}$, for the FSI of the $I=\frac{1}{2}$ member of the octet. The phases $\delta_{0}, \delta_{0}^{\prime}, \delta_{1}$ and $\delta_{\frac{1}{2}}$ and the mixing angle $\phi$ are defined such that in the limit of $\mathrm{SU}(3)_{F}$ conservation $\delta_{0}=\delta_{1}=\delta_{\frac{1}{2}}$, the amplitudes are independent of $\delta_{0}^{\prime}$ and $\phi=\pi / 2$. The phase for the decay modes with $D_{s}^{+}$in the initial state is expected to be different from those in the $D^{0}$ and $D^{+}$decay modes as an effect of $\mathrm{SU}(3)_{F}$ breaking and the consequent shift in the mass of the $D_{s}^{+}$. Keeping in mind that both the phases shift in the same direction, we parametrize the phases with $\epsilon_{\delta}$ as

$$
\delta_{1}^{\prime}=\delta_{1}\left(1-\epsilon_{\delta}\right) \quad \text { and } \quad \delta_{\frac{1}{2}}^{\prime}=\delta_{\frac{1}{2}}\left(1-\epsilon_{\delta}\right) .
$$

The extension to the CA and DCS final states requires the introduction of additional sources of $\mathrm{SU}(3)_{F}$ violation in addition to $\delta_{\frac{1}{2}}$ in the $I=\frac{1}{2}$ octet channel. To understand this better one must note that the $\mathrm{SU}(3)_{F}$ relationship for $D^{+}$decays:

$$
\tan \theta_{C} A\left(D^{+} \rightarrow \overline{K^{0}} \pi^{+}\right)=\sqrt{2} A\left(D^{+} \rightarrow \pi^{0} \pi^{+}\right),
$$

which implies (neglecting the interference with the DCS final state) the ratio of the decay amplitudes into two pions and into $K_{S} \pi^{+}$being equal to $\tan \theta_{C}$ is in disagreement with data. To correct for this discrepancy we allow for a breaking of the 27 amplitudes through the introductions of a parameters $\kappa$ and $\kappa^{\prime}$ which, respectively, split the 27 matrix element in the CA and DCS channels from the 27 matrix element in the SCS channel.

Next, we observe that the ratio of the branching fractions of the DCS to the CA decays of $D^{0}$ into a kaon and a pion with opposite electric charge given by

$$
\frac{\mathrm{BR}\left(D^{0} \rightarrow K^{+} \pi^{-}\right)}{\operatorname{BR}\left(D^{0} \rightarrow K^{-} \pi^{+}\right)}=\tan ^{4} \theta_{C}
$$

is violated and the ratio is actually larger than the value $\tan ^{4} \theta_{C}$. To accommodate for this we allow for the $\mathrm{SU}(3)_{F}$ breaking parameter $K$ which contributes with opposite signs to the octet part for the CA and DCS channels to correct the prediction in Eq. (5). This parameter represents the nonconservation of the strangeness changing current which, in the factorization ansatz, corresponds to the axial current that destroys the initial $D$ meson state and the divergence in the vector current proportional to the mass difference of the strange quark and that of the lighter quarks $u$ and $d$. This also generates a term proportional to $K^{\prime}$ in the DCS decays of the $D^{+}$and the CA decays of the $D_{s}^{+}$meson which comes from the fact that for annihilating the charged mesons a charged current is necessary.

With this parametrization we arrive at the following amplitudes for the SCS, CA and DCS amplitudes. Although we present the amplitudes with $\eta_{8}$ in the final state, we do not make any attempt to include $\eta-\eta^{\prime}$ mixing in this work and hence do not use the experimental measurements of these channels for the fits. While considering the singlet state, $\eta_{1}$, would increase the number of measurements that we could fit the parameters to, including the singlet state would also require additional parameters since it is has a significant gluonic content $[93,94]$. A discussion of the complexities of addressing $\eta-\eta^{\prime}$ mixing can be found in [95] and references therein. Hence, we shall postpone this exercise to a future work.

In summary, the $\mathrm{SU}(3)_{F}$ breaking parameters that we need to introduce to fit to the $\Delta U=1$ part of the amplitudes that are sensitive to the measurements of the branching fraction are as follows:

(1) For the phases generated by FSI in the different isospin amplitudes:

(a) $\delta_{0}$ : the FSI phase of the singlet component of the $I=0$ amplitude;

(b) $\delta_{0}^{\prime}$ : the FSI phase of the octet component of the $I=0$ amplitude;

(c) $\delta_{\frac{1}{2}}$ : the FSI phace of the $I=1 / 2$ amplitude;

(d) $\epsilon_{\delta}^{2}$ : Defined in Eq. (3) as the splitting in the phase of $I=0$ amplitudes for the heavier $D_{s}^{+}$meson from the lighter $D^{0}$ and $D^{+}$mesons.

(2) A mixing angle $\phi$ that characterizes the mixing between the singlet and octet components in the $I=0$ amplitude.

(3) $K$ and $K^{\prime}$ : Comes from the nonconservation of the strangeness changing neutral and charged currents respectively and accommodates for the deviation of data from Eq. (5).

(4) $\kappa$ and $\kappa^{\prime}$ : Introduced to allow for $\mathrm{SU}(3)_{F}$ breaking in the 27 matrix element to alleviate the discrepancy in Eq. (4) for the CA and DCS amplitudes respectively. The $\Delta U=1$ amplitudes in terms of these parameters can be written as follows:

SCS modes [to be multiplied by $\frac{1}{2}\left(V_{c s} V_{u s}^{*}-V_{c d} V_{u d}^{*}\right)$ ]

$$
\begin{aligned}
A\left(D^{0} \rightarrow \pi^{+} \pi^{-}\right) & =\left(T-\frac{2}{3} C\right)\left[-\frac{3}{10}\left(e^{i \delta_{0}}+e^{i \delta_{0}^{\prime}}\right)+\left(-\frac{3}{10} \cos (2 \phi)+\frac{3}{4 \sqrt{10}} \sin (2 \phi)\right)\left(e^{i \delta_{0}^{\prime}}-e^{i \delta_{0}}\right)\right]-\frac{2}{5}(T+C) \\
A\left(D^{0} \rightarrow \pi^{0} \pi^{0}\right) & =\left(T-\frac{2}{3} C\right)\left[-\frac{3}{10}\left(e^{i \delta_{0}}+e^{i \delta_{0}^{\prime}}\right)+\left(-\frac{3}{10} \cos (2 \phi)+\frac{3}{4 \sqrt{10}} \sin (2 \phi)\right)\left(e^{i \delta_{0}^{\prime}}-e^{i \delta_{0}}\right)\right]+\frac{3}{5}(T+C) \\
A\left(D^{0} \rightarrow K^{+} K^{-}\right) & =\left(T-\frac{2}{3} C\right)\left[\frac{3}{20}\left(e^{i \delta_{0}}+e^{i \delta_{0}^{\prime}}\right)+\left(\frac{3}{20} \cos (2 \phi)+\frac{3}{4 \sqrt{10}} \sin (2 \phi)\right)\left(e^{i \delta_{0}^{\prime}}-e^{i \delta_{0}}\right)+\frac{3}{10} e^{i \delta_{1}}\right]+\frac{2}{5}(T+C)
\end{aligned}
$$




$$
\begin{aligned}
A\left(D^{0} \rightarrow K^{0} \bar{K}^{0}\right) & =\left(T-\frac{2}{3} C\right)\left[\frac{3}{20}\left(e^{i \delta_{0}}+e^{i \delta_{0}^{\prime}}\right)+\left(\frac{3}{20} \cos (2 \phi)+\frac{3}{4 \sqrt{10}} \sin (2 \phi)\right)\left(e^{i \delta_{0}^{\prime}}-e^{i \delta_{0}}\right)-\frac{3}{10} e^{i \delta_{1}}\right] \\
A\left(D^{0} \rightarrow \eta_{8} \eta_{8}\right) & =\left(T-\frac{2}{3} C\right)\left[\frac{3}{10}\left(e^{i \delta_{0}}+e^{i \delta_{0}^{\prime}}\right)+\left(\frac{3}{10} \cos (2 \phi)+\frac{3}{4 \sqrt{10}} \sin (2 \phi)\right)\left(e^{i \delta_{0}^{\prime}}-e^{i \delta_{0}}\right)\right]-\frac{3}{5}(T+C) \\
A\left(D^{0} \rightarrow \pi^{0} \eta_{8}\right) & =\frac{\sqrt{3}}{5}\left[\left(T-\frac{2}{3} C\right) e^{i \delta_{1}}-(T+C)\right] \\
A\left(D^{+} \rightarrow K^{+} \bar{K}^{0}\right) & =\frac{1}{5}(2 T-3 C+\Delta) e^{i \delta_{1}}+\frac{3}{5}(T+C) \\
A\left(D^{+} \rightarrow \pi^{+} \pi^{0}\right) & =\frac{1}{\sqrt{2}}(T+C) \\
A\left(D^{+} \rightarrow \pi^{+} \eta_{8}\right) & =\frac{\sqrt{2}}{5 \sqrt{3}}(2 T-3 C+\Delta) e^{i \delta_{1}}-\frac{3 \sqrt{3}}{5 \sqrt{2}}(T+C) \\
A\left(D_{s}^{+} \rightarrow \pi^{+} K^{0}\right) & =-\frac{1}{5}\left(2 T-3 C+\Delta-K^{\prime}\right) e^{i \delta_{1}^{\prime}}-\frac{3}{5}(T+C) \\
A\left(D_{s}^{+} \rightarrow \pi^{0} K^{+}\right) & =-\frac{1}{5 \sqrt{2}}\left(2 T-3 C+\Delta-K^{\prime}\right) e^{i \delta_{\frac{1}{2}}^{\prime}}+\frac{2}{5 \sqrt{2}}(T+C) \\
A\left(D_{s}^{+} \rightarrow K^{+} \eta_{8}\right) & =\frac{1}{5 \sqrt{6}}\left(2 T-3 C+\Delta-K^{\prime}\right) e^{i \delta_{\frac{1}{2}}^{\prime}}-\frac{2 \sqrt{6}}{5}(T+C)
\end{aligned}
$$

CA modes [to be multiplied by $V_{c s} V_{u d}^{*}$ ]

$$
\begin{aligned}
A\left(D^{0} \rightarrow \pi^{+} K^{-}\right) & =\frac{1}{5}(3 T-2 C-K) e^{i \delta_{\frac{1}{2}}}+\frac{2}{5}(T+C+\kappa) \\
A\left(D^{0} \rightarrow \pi^{0} \bar{K}^{0}\right) & =-\frac{1}{5 \sqrt{2}}(3 T-2 C-K) e^{i \delta_{\frac{1}{2}}}+\frac{3}{5 \sqrt{2}}(T+C+\kappa) \\
A\left(D^{0} \rightarrow \bar{K}^{0} \eta_{8}\right) & =-\frac{1}{5 \sqrt{6}}(3 T-2 C-K) e^{i \delta_{\frac{1}{2}}}+\frac{3}{5 \sqrt{6}}(T+C+\kappa) \\
A\left(D^{+} \rightarrow \pi^{+} \bar{K}^{0}\right) & =(T+C+\kappa) \\
A\left(D_{s}^{+} \rightarrow K^{+} \bar{K}^{0}\right) & =-\frac{1}{5}(2 T-3 C+\Delta) e^{i \delta_{1}^{\prime}}+\frac{2}{5}(T+C+\kappa) \\
A\left(D_{s}^{+} \rightarrow \pi^{+} \eta_{8}\right) & =-\frac{\sqrt{2}}{5 \sqrt{3}}(2 T-3 C+\Delta) e^{i \delta_{1}^{\prime}}-\frac{\sqrt{6}}{5}(T+C+\kappa)
\end{aligned}
$$

DCS modes [to be multiplied by $-V_{c d} V_{u s}^{*}$ ]

$$
\begin{aligned}
A\left(D^{0} \rightarrow \pi^{0} K^{0}\right) & =\frac{1}{5 \sqrt{2}}(3 T-2 C+K) e^{i \delta_{\frac{1}{2}}}-\frac{3}{5 \sqrt{2}}\left(T+C+\kappa^{\prime}\right) \\
A\left(D^{0} \rightarrow K^{0} \eta_{8}\right) & =\frac{1}{5 \sqrt{6}}(3 T-2 C+K) e^{i \delta_{\frac{1}{2}}}-\frac{3}{5 \sqrt{6}}\left(T+C+\kappa^{\prime}\right) \\
A\left(D^{+} \rightarrow \pi^{+} K^{0}\right) & =\frac{1}{5}\left(2 T-3 C+\Delta-K^{\prime}\right) e^{i \delta_{\frac{1}{2}}}-\frac{2}{5}\left(T+C+\kappa^{\prime}\right) \\
A\left(D^{0} \rightarrow \pi^{-} K^{+}\right) & =-\frac{1}{5}(3 T-2 C+K) e^{i \delta_{\frac{1}{2}}}-\frac{2}{5}\left(T+C+\kappa^{\prime}\right) \\
A\left(D^{+} \rightarrow \pi^{0} K^{+}\right) & =\frac{1}{5 \sqrt{2}}\left(2 T-3 C+\Delta-K^{\prime}\right) e^{i \delta_{\frac{1}{2}}}+\frac{3}{5 \sqrt{2}}\left(T+C+\kappa^{\prime}\right) \\
A\left(D^{+} \rightarrow K^{+} \eta_{8}\right) & =-\frac{1}{5 \sqrt{6}}\left(2 T-3 C+\Delta-K^{\prime}\right) e^{i \delta_{\frac{1}{2}}}-\frac{3}{5 \sqrt{6}}\left(T+C+\kappa^{\prime}\right) \\
A\left(D_{s}^{+} \rightarrow K^{+} K^{0}\right) & =-\left(T+C+\kappa^{\prime}\right) .
\end{aligned}
$$


The CKM factors are to be kept explicit and hence the amplitudes given in Eqs. (6)-(8) should be multiplied by $\frac{1}{2}\left(V_{u s} V_{c s}^{*}-V_{u d} V_{c d}^{*}\right), V_{u d} V_{c s}^{*}$ and $-V_{u s} V_{c d}^{*}$ for SCS, CA, and DCS modes, respectively. The branching fraction is then defined as

$$
\operatorname{BR}\left(D \rightarrow P_{1} P_{2}\right)=\frac{\tau_{D} G_{F}^{2}}{16 \pi m_{D}^{2}} \frac{\sqrt{\left(m_{D}^{2}-\left(m_{P_{1}}+m_{P_{2}}\right)^{2}\right)\left(m_{D}^{2}-\left(m_{P_{1}}-m_{P_{2}}\right)^{2}\right)}}{2 m_{D}} \times\left|\mathcal{A}\left(D \rightarrow P_{1} P_{2}\right)\right|^{2},
$$

where $m_{D}, m_{P_{1}}$, and $m_{P_{2}}$ are the masses of the $D$ meson in the initial state and the pseudoscalars in the final state respectively, $G_{F}$ is the Fermi constant, and $\tau_{D}$ is the relevant $D$ meson lifetime.

\section{THE $\Delta U=0$ AMPLITUDES}

The $\Delta U=0$ contributions to the SCS decays proportional to $V_{c b} V_{u b}^{*}$ need to be considered both for the amplitudes related to the penguin operator:

$$
\begin{aligned}
& \bar{u}_{L}(x) \gamma^{\mu} \lambda_{a} c_{L}(x)\left[\left(\bar{u}(x) \gamma_{\mu} \lambda_{a} u(x)\right.\right. \\
& \left.\quad+\bar{d}(x) \gamma^{\mu} \lambda_{a} d(x)+\bar{s}(x) \gamma_{\mu} \lambda_{a} s(x)\right]
\end{aligned}
$$

and to the operator:

$$
\overline{u_{L}} \gamma_{\mu} s_{L} \bar{s}_{L} \gamma^{\mu} c_{L}(x)+\overline{u_{L}} \gamma_{\mu} d_{L} \overline{d_{L}} \gamma^{\mu} c_{L}(x) \text {. }
$$

The latter have to be considered as a consequence of the unitarity of the CKM matrix and are referred to as the pseudopenguin operators. Parametrizing this part of the amplitude requires the introduction of three additional real parameters $P, \Delta_{3}$, and $\Delta_{4}$. The penguin contributions are encapsulated in $P$. The matrix elements of the operator defined in Eq. (11) depend on four reduced matrix elements, $\langle 27|15| \overline{3}\rangle,\langle 8|15| \overline{3}\rangle$, and $\langle 1|3| \overline{3}\rangle$. The first two are related to the ones for the $\Delta U=1$ part. We introduce two parameters, $\Delta_{3}$ and $\Delta_{4}$, which are combinations of the four reduced matrix elements which are defined in such a way that, by neglecting final state interactions, one has

$$
\begin{aligned}
B\left(D^{0} \rightarrow K^{+} K^{-}\right) & =P+T+\Delta_{3} \equiv \mathcal{P}, \\
B\left(D^{0} \rightarrow K^{0} \bar{K}^{0}\right) & =\Delta_{4} .
\end{aligned}
$$

The asymmetries consist of three contributions. The first contribution comes from the terms proportional to $\mathcal{P}$. While $T$ can be extracted from the branching fraction data neither $\mathcal{P}$ nor $\Delta_{3}$ can be estimated from first principles. The second contribution is proportional to $T+C$ and it can be completely determined from the branching fraction data. The third contribution is proportional to $\Delta_{4}$. This contribution, which is a sum of penguin and pseudopenguin contributions, is vanishingly small due to an approximate selections rule which disfavors the simultaneous creation of $d \bar{d}$ and $s \bar{s}$ pairs [96]. Moreover, $\mathrm{SU}(3)_{F}$ relates the $\Delta$ in the $\Delta U=1$ part to the $\Delta_{4}$ in the $\Delta U=0$ part. It is interesting to note here that indeed the $\Delta U=0$ contributions of the 15 would allow a contribution to $D^{0} \rightarrow K^{0} \bar{K}^{0}$. To forbid it, according to the selection rule, one should put $\Delta \sim 0$. The fact that the fit to the branching ratios implies for $\Delta$ a small value consistent with 0 lies in favor of the selection rule. However $\Delta_{3}$ is not affected by this approximate selection rule and hence does not need to be vanishingly small. There is a contribution proportional to $\Delta$ in the $\Delta U=0$ part of the amplitudes of the $D^{+}$modes. However, as we shall see, $\Delta$ is very small and hence this contribution turns out to be insignificant. The explicit forms of the $\Delta U=0$ part of the amplitude are as follows:

$$
\begin{aligned}
B\left(D^{0} \rightarrow \pi^{+} \pi^{-}\right)= & \mathcal{P}\left(\frac{1}{2}\left(e^{i \delta_{0}^{\prime}}+e^{i \delta_{0}}\right)+\left(e^{i \delta_{0}^{\prime}}-e^{i \delta_{0}}\right)\left(-\frac{1}{6} \cos (2 \phi)-\frac{7}{4 \sqrt{10}} \sin (2 \phi)\right)\right) \\
& +(T+C)\left(-\frac{3}{20}\left(e^{i \delta_{0}^{\prime}}+e^{i \delta_{0}}\right)+\frac{3}{10}+\left(\frac{1}{60} \cos (2 \phi)+\frac{1}{2 \sqrt{10}} \sin (2 \phi)\right)\left(e^{i \delta_{0}^{\prime}}-e^{i \delta_{0}}\right)\right) \\
& +\Delta_{4}\left(e^{i \delta_{0}^{\prime}}-e^{i \delta_{0}}\right)\left(-\frac{1}{3} \cos (2 \phi)-\frac{1}{4 \sqrt{10}} \sin (2 \phi)\right) \\
B\left(D^{0} \rightarrow \pi^{0} \pi^{0}\right)= & B\left(D^{0} \rightarrow \pi^{+} \pi^{-}\right)-(T+C), \\
B\left(D^{0} \rightarrow K^{+} K^{-}\right)= & \mathcal{P}\left(\frac{1}{4}\left(e^{i \delta_{0}^{\prime}}+e^{i \delta_{0}}\right)+\left(e^{i \delta_{0}^{\prime}}-e^{i \delta_{0}}\right)\left(-\frac{5}{12} \cos (2 \phi)+\frac{1}{4 \sqrt{10}} \sin (2 \phi)\right)+\frac{1}{2} e^{i \delta_{1}}\right) \\
& +(T+C)\left(-\frac{1}{20}\left(e^{i \delta_{0}^{\prime}}+e^{i \delta_{0}}\right)+\frac{3}{10}+\frac{7}{60} \cos (2 \phi)\left(e^{i \delta_{0}^{\prime}}-e^{i \delta_{0}}\right)-\frac{1}{5} e^{i \delta_{1}}\right) \\
& +\Delta_{4}\left(\frac{1}{4}\left(e^{i \delta_{0}^{\prime}}+e^{i \delta_{0}}\right)+\left(e^{i \delta_{0}^{\prime}}-e^{i \delta_{0}}\right)\left(-\frac{1}{12} \sin (2 \phi)+\frac{3}{4 \sqrt{10}} \sin (2 \phi)\right)-\frac{1}{2} e^{i \delta_{1}}\right)
\end{aligned}
$$




$$
\begin{aligned}
& B\left(D^{0} \rightarrow K^{0} \bar{K}^{0}\right)=\mathcal{P}\left(\frac{1}{4}\left(e^{i \delta_{0}^{\prime}}+e^{i \delta_{0}}\right)+\left(e^{i \delta_{0}^{\prime}}-e^{i \delta_{0}}\right)\left(-\frac{5}{12} \cos (2 \phi)+\frac{1}{4 \sqrt{10}} \sin (2 \phi)\right)-\frac{1}{2} e^{i \delta_{1}}\right) \\
& +(T+C)\left(-\frac{1}{20}\left(e^{i \delta_{0}^{\prime}}+e^{i \delta_{0}}\right)-\frac{1}{10}+\frac{7}{60} \cos (2 \phi)\left(e^{i \delta_{0}^{\prime}}-e^{i \delta_{0}}\right)+\frac{1}{5} e^{i \delta_{1}}\right) \\
& +\Delta_{4}\left(\frac{1}{4}\left(e^{i \delta_{0}^{\prime}}+e^{i \delta_{0}}\right)+\left(e^{i \delta_{0}^{\prime}}-e^{i \delta_{0}}\right)\left(-\frac{1}{12} \cos (2 \phi)+\frac{3}{4 \sqrt{10}} \sin (2 \phi)\right)+\frac{1}{2} e^{i \delta_{1}}\right), \\
& B\left(D^{0} \rightarrow \pi^{0} \eta_{8}\right)=\frac{1}{\sqrt{3}}\left(\left(\mathcal{P}-\Delta_{4}\right) e^{i \delta_{1}}-(T+C)\left(\frac{2}{5} e^{i \delta_{1}}+\frac{3}{5}\right)\right), \\
& B\left(D^{+} \rightarrow K^{+} \bar{K}^{0}\right)=\left(\mathcal{P}-\Delta_{4}-\frac{1}{5}(\Delta+T+C)\right) e^{i \delta_{1}}+\frac{1}{5}(T+C), \\
& B\left(D^{+} \rightarrow \pi^{+} \eta_{8}\right)=\sqrt{\frac{2}{3}}\left(\mathcal{P}-\Delta_{4}-\frac{1}{5}(\Delta+T+C)\right) e^{i \delta_{1}}-\frac{\sqrt{6}}{10}(T+C), \\
& B\left(D_{s}^{+} \rightarrow K^{0} \pi^{+}\right)=-\left(\mathcal{P}-\Delta_{4}-\frac{1}{5}(\Delta+T+C)\right) e^{i \delta^{\prime}} \frac{1}{2}-\frac{1}{5}(T+C), \\
& B\left(D_{s}^{+} \rightarrow K^{+} \pi^{0}\right)=-\sqrt{\frac{1}{2}}\left(\mathcal{P}-\Delta_{4}-\frac{1}{5}(\Delta+T+C)\right) e^{i \delta_{1}^{\prime}}+\frac{2 \sqrt{2}}{5}(T+C), \\
& B\left(D_{s}^{+} \rightarrow K^{+} \eta_{8}\right)=\sqrt{\frac{1}{6}}\left(\mathcal{P}-\Delta_{4}-\frac{1}{5}(\Delta+T+C)\right) e^{i \delta_{\frac{1}{2}}^{\prime}}+\frac{\sqrt{6}}{5}(T+C) .
\end{aligned}
$$

The total amplitude for the SCS decays where we consider $C P$ violation can now be written as

$$
\begin{aligned}
\mathcal{A}\left(D \rightarrow P_{1} P_{2}\right)= & \frac{1}{2}\left[\left(V_{u s} V_{c s}^{*}-V_{u d} V_{c d}^{*}\right) A\left(D \rightarrow P_{1} P_{2}\right)\right. \\
& \left.+\left(V_{u s} V_{c s}^{*}+V_{u d} V_{c d}^{*}\right) B\left(D \rightarrow P_{1} P_{2}\right)\right]
\end{aligned}
$$

with $P_{1}$ and $P_{2}$ as $\pi, K$, or $\eta_{8}$.

The $\Delta U=0$ part of the $D^{0} \rightarrow K_{S} K_{S}$ decay amplitude includes a weak exchange topology. This contribution can be potentially large and lead to the enhancement of the $C P$ asymmetry in this channel $[83,100]$ due to the Zweig suppression of the $\Delta U=1$ part of the amplitude. It has been pointed out in [83] that the possibly large weak exchange contribution and the suppression of the branching fraction decorrelates the $C P$ asymmetry in this channel from the other SCS channels where the weak exchange topology does not contribute. In our framework, this weak exchange topology is generated by rescattering and hence related to the parameters in the $\Delta U=1$ amplitude. The generation of the weak exchange topology by rescattering was also discussed in [101]. This characterization of the weak exchange topology leads to the parametric correlation between all the $\operatorname{SCS} \Delta U=0$ amplitudes leading to a correlation amongst the $C P$ violation in these channels.

\section{MEASUREMENTS OF $C P$ ASYMMETRIES}

Much progress has been made in the measurement of $C P$ asymmetries, a compendium of which can be found on the HFLAV [102] website. It is important to note here that the $C P$ asymmetries measured by the experiments in the neutral $D^{0}$ channel is the sum of direct and indirect $C P$ asymmetries (time integrated), while the HFLAV averages are direct $C P$ asymmetries only. For the decay of the charged $D$ mesons the direct $C P$ asymmetry is measured. The most notable of the $C P$ asymmetry measurements is the very precise measurement of $\Delta \mathrm{A}_{C P}=$ $\mathrm{A}_{C P}\left(D^{0} \rightarrow K^{+} K^{-}\right)-\mathrm{A}_{C P}\left(D^{0} \rightarrow \pi^{+} \pi^{-}\right)$by LHCb [45] with their 7 and $8 \mathrm{TeV}$ data. Combining this result with the previous $\mathrm{LHCb}$ measurement [44] and the $\mathrm{LHCb}$ measurement of indirect asymmetry [43,103] using $A_{\Gamma} \sim-\Delta \mathrm{A}_{C P}^{\text {ind }}$ and the measurement of $y_{\mathrm{CP}}$ [48] they extracted the difference of the direct $C P$ asymmetry as

$$
\Delta \mathrm{A}_{C P}^{\operatorname{dir}}=(-0.061 \pm 0.076) \%
$$

while the HFLAV world average stood at [102]

$$
\Delta \mathrm{A}_{C P}^{\mathrm{dir}}=(-0.137 \pm 0.070) \% .
$$

Recently, $\mathrm{LHCb}$ has released the analysis of $\Delta \mathrm{A}_{C P}$ with combined $9 \mathrm{fb}^{-1}$ of data collected over Run I and II [104]. With this analysis, the collaboration has measured $\Delta \mathrm{A}_{C P}$ with more than $5 \sigma$ significance. This is the first measurement of $C P$ asymmetry in the up-type quark sector and the only significant measurement of $C P$ asymmetry in charm mesons. Considering the relevance of this measurement, we include this in our analysis even though it was released after the first version of our work was made public. From the 
TABLE I. Measurements of $C P$ asymmetries in various channels.

\begin{tabular}{lcl}
\hline \hline Channel & Mean \pm rms (\%) & \multicolumn{1}{c}{ Reference } \\
\hline$D^{0} \rightarrow K^{+} K^{-}$ & $-0.16 \pm 0.12$ & HFLAV [102] \\
$D^{0} \rightarrow \pi^{+} \pi^{-}$ & $0.00 \pm 0.15$ & HFLAV [102] \\
$D^{0} \rightarrow \pi^{0} \pi^{0}$ & $-0.03 \pm 0.64$ & HFLAV [105,106] \\
$D^{+} \rightarrow K^{+} K_{S}$ & $-0.11 \pm 0.25$ & HFLAV [107-110] \\
$D_{s}^{+} \rightarrow K_{S} \pi^{+}$ & $0.38 \pm 0.48$ & HFLAV [102] \\
$D_{s}^{+} \rightarrow K^{+} \pi^{0}$ & $-0.266 \pm 0.238 \pm 0.009$ & CLEO [108] \\
$D^{0} \rightarrow K_{S} K_{S}$ & $-2.9 \pm 5.2 \pm 2.2$ & LHCb [111] \\
$D^{0} \rightarrow K_{S} K_{S}$ & $-0.2 \pm 1.53 \pm 0.17$ & Belle [112] \\
\hline \hline
\end{tabular}

$\mathrm{LHCb}$ measurements we have

$$
\begin{aligned}
& \Delta \mathrm{A}_{C P}=(-0.154 \pm 0.029) \%, \\
& \Delta \mathrm{A}_{C P}^{\mathrm{dir}}=(-0.156 \pm 0.029) \%,
\end{aligned}
$$

which leads to a world average of

$$
\Delta \mathrm{A}_{C P}^{\mathrm{dir}}=(-0.164 \pm 0.028) \% .
$$

We do not use the measurement of the individual asymmetries $\mathrm{A}_{C P}\left(D^{0} \rightarrow \pi^{+} \pi^{-}\right)$and $\mathrm{A}_{C P}\left(D^{0} \rightarrow K^{+} K^{-}\right)$ since the LHCb results for these [44] are used in the estimation of $\Delta \mathrm{A}_{C P}^{\mathrm{dir}}$. The results from the other experiments on these individual asymmetries do not improve the fit in any manner since they are much less precise. We have numerically checked the validity of this statement. For the sake of completeness we list some of the relevant $C P$ asymmetries in Table I that have been measured till date. We do not use these measurements in the fit but predict them from a fit to the branching fractions and the HFLAV average of $\Delta \mathrm{A}_{C P}^{\mathrm{dir}}$.

In the recent past some theoretical effort has been put on estimating $C P$ asymmetry in $D^{0} \rightarrow K_{S} K_{S}[73,76,83]$ along with experimental measurements being performed at $\mathrm{LHCb}$ [111] and Belle [112] as listed in Table I. There is an older measurement by CLEO [105] which we do not quote here since it is much less precise. In [73], $\mathrm{A}_{C P}\left(D^{0} \rightarrow K_{S} K_{S}\right)$ was estimated to be about $0.6 \%$ in magnitude. In [76] the $C P$ asymmetry in $D^{0} \rightarrow K_{S} K_{S}$ was related to $\Delta \mathrm{A}_{C P}^{\text {dir }}$ and an estimation of about $0.4 \%$ was made for the former. In [83] it was shown that this asymmetry can be of $O(1 \%)$ due to possibly large contributions from the weak exchange diagrams to the $\Delta U=0$ part of the amplitude. In the following section we present our results for $A_{C P}\left(D^{0} \rightarrow K_{S} K_{S}\right)$ using data on both branching fractions and $\Delta \mathrm{A}_{C P}^{\mathrm{dir}}$ to constrain the parameters along with the prediction of $C P$ asymmetries of several other SCS modes.

The only SCS channel for which the $C P$ asymmetry is predictably 0 in the SM is that in $D^{+} \rightarrow \pi^{+} \pi^{0}[113,114]$ since it is driven by a single isospin amplitude and hence lacks the two separate strong and weak phases necessary for a nonzero $C P$ asymmetry. Recently Belle has measured a $C P$ asymmetry in this channel consistent with the null SM value [115]:

$$
\mathrm{A}_{C P}\left(D^{+} \rightarrow \pi^{+} \pi^{0}\right)=(2.31 \pm 1.24 \pm 0.23) \%
$$

which has a much better precision than the previous CLEO measurement [108] which has a error of $2.9 \%$ and is consistent with the null SM value.

The BESIII Collaboration has performed the first measurements of $C P$ asymmetry in $D^{+} \rightarrow K^{+} K_{S}$ and $D^{+} \rightarrow$ $K^{+} K_{L}$ [116] which should be exactly equal since both are driven by $D^{+} \rightarrow K^{+} \bar{K}^{0}$ only. The two measurements are in good agreement with each other and consistent with 0 :

$$
\begin{aligned}
& \mathrm{A}_{C P}\left(D^{+} \rightarrow K^{+} K_{S}\right)=(-1.8 \pm 2.7 \pm 1.6) \%, \\
& \mathrm{~A}_{C P}\left(D^{+} \rightarrow K^{+} K_{L}\right)=(-4.2 \pm 3.2 \pm 1.2) \% .
\end{aligned}
$$

\section{RESULTS AND THEIR CONSEQUENCES}

We use HEPFIT [117] to perform a fit in the Bayesian framework. The 7 amplitudes $\left(T, C, \Delta, K, K^{\prime}, \kappa\right.$, and $\left.\kappa^{\prime}\right)$, the $\mathrm{SU}(3)_{F}$ breaking parameter quantifying the shift in the $D_{s}^{+}$ phase $\left(\epsilon_{\delta}\right), 4$ phases $\left(\delta_{0}, \delta_{0}^{\prime}, \delta_{\frac{1}{2}}, \delta_{1}\right)$, and a mixing angle $\phi$ are constrained using 17 branching fractions. The $\Delta U=0$ part of the amplitudes requires 3 additional parameters $P, \Delta_{3}$,

TABLE II. The branching fractions that were used in the fit $[116,118,119]$. We also use $\operatorname{BR}\left(D_{s}^{+} \rightarrow K^{+} K_{S, L}\right)=(29.5 \pm 1.4) \times$ $10^{-3}$ [120] measured by Belle. The fit results are the same for both the negative and the positive solutions for the phases, $\delta_{i}$.

\begin{tabular}{lccc}
\hline \hline Channel & Fit $\left(\times 10^{-3}\right)$ & PDG $\left(\times 10^{-3}\right)$ & BESIII $\left(\times 10^{-3}\right)$ \\
\hline \multicolumn{4}{c}{ SCS } \\
$D^{0} \rightarrow \pi^{+} \pi^{-}$ & $01.448 \pm 0.019$ & $1.407 \pm 0.025$ & $1.508 \pm 0.028$ \\
$D_{0}^{+} \rightarrow \pi^{0} \pi^{0}$ & $00.816 \pm 0.025$ & $0.822 \pm 0.025$ & $\ldots$ \\
$D^{+} \rightarrow \pi^{+} \pi^{0}$ & $01.235 \pm 0.033$ & $1.17 \pm 0.06$ & $1.259 \pm 0.040$ \\
$D^{0} \rightarrow K^{+} K^{-}$ & $04.064 \pm 0.044$ & $3.97 \pm 0.07$ & $4.233 \pm 0.067$ \\
$D^{0} \rightarrow K_{S} K_{S}$ & $00.168 \pm 0.012$ & $00.17 \pm 0.012$ & $\ldots$ \\
$D^{+} \rightarrow K^{+} K_{S}$ & $03.164 \pm 0.056$ & $2.83 \pm 0.16$ & $3.183 \pm 0.067$ \\
$D^{+} \rightarrow K^{+} K_{L}$ & $03.164 \pm 0.056$ & $\ldots$ & $3.21 \pm 0.16$ \\
$D_{s}^{+} \rightarrow \pi^{0} K^{+}$ & $01.41 \pm 0.15$ & $0.63 \pm 0.21$ & $\cdots$ \\
$D_{s}^{+} \rightarrow \pi^{+} K_{S}$ & $01.24 \pm 0.06$ & $1.22 \pm 0.06$ & $\cdots$ \\
\multicolumn{4}{c}{$\mathrm{CA}$ and DCS } \\
$D^{+} \rightarrow \pi^{+} K_{S}$ & $15.80 \pm 0.29$ & $14.7 \pm 0.8$ & $15.91 \pm 0.31$ \\
$D^{+} \rightarrow \pi^{+} K_{L}$ & $14.37 \pm 0.52$ & $14.6 \pm 0.5$ & $\cdots$ \\
$D^{0} \rightarrow \pi^{+} K^{-}$ & $38.96 \pm 0.32$ & $38.9 \pm 0.4$ & $\ldots$ \\
$D^{0} \rightarrow \pi^{0} K_{S}$ & $12.29 \pm 0.21$ & $11.9 \pm 0.4$ & $12.39 \pm 0.28$ \\
$D^{0} \rightarrow \pi^{0} K_{L}$ & $09.73 \pm 0.21$ & $10.0 \pm 0.7$ & $\ldots$ \\
$D_{s}^{+} \rightarrow K^{+} K_{S}$ & $14.67 \pm 0.41$ & $15.0 \pm 0.5$ & $\ldots$ \\
$D^{+} \rightarrow \pi^{0} K^{+}$ & $00.151 \pm 0.013$ & $00.181 \pm 0.027$ & $0.231 \pm 0.022$ \\
$D^{0} \rightarrow \pi^{-} K^{+}$ & $00.141 \pm 0.003$ & $00.1385 \pm 0.0027$ & $\ldots$ \\
$D^{0} \rightarrow \pi^{ \pm} K^{\mp}$ & $39.1 \pm 0.32$ & $\cdots$ & $38.98 \pm 0.52$ \\
\hline \hline
\end{tabular}


and $\Delta_{4}$. The first two, $P$ and $\Delta_{3}$ always appear as a sum in the $\Delta U=0$ part of all the decay amplitudes and hence it is not possible to disentangle them individually from data. Moreover, it is not possible to estimate the sizes of these parameters from first principles and hence we work with the ratio $\left(P+\Delta_{3}\right) / T$ in our fit and use $\Delta \mathrm{A}_{C P}^{\mathrm{dir}}$ to constrain this combination. As discussed before, $\Delta_{4}$ is expected to be tiny due to the approximate selection rule and so we set it to 0 in our fit. The experimental numbers used for the fit are listed in Table II and in Eq. (15). For the branching fractions we use the $D^{0}$ and the $D_{(s)}^{+}$decays with only $\pi$ and $K$ in the final state. In addition to the PDG averages listed in Table II we also use the recent measurements made by the BESIII Collaboration [116,118] which are comparable or better than the PDG averages.

\section{A. Fit to branching fractions and $\Delta \mathrm{A}_{C P}^{\mathrm{dir}}$}

The fit results are presented in Table III along with the correlation matrix for the fitted parameters. The parameter $\left(P+\Delta_{3}\right) / T$ is excluded from the correlation matrix because it is essentially uncorrelated with the other parameters being determined by $\Delta \mathrm{A}_{C P}^{\mathrm{dir}}$ while the other parameters are determined by the branching ratio data. As a cross-check we also performed a fit using MINUIT routines and have verified that the results are exactly the same. The error analysis was done in HEPFIT and is taken as the rms of the posterior distributions of the parameters and observables.

We find two equivalent solutions for the parameters in the $\Delta U=1$ part of the amplitude from the branching fractions alone. The solutions are distinct only for the posterior distributions of the 4 phases $\left(\delta_{0}, \delta_{0}^{\prime}, \delta_{\frac{1}{2}}, \delta_{1}\right)$ with $\delta_{i} \rightarrow-\delta_{i}$ relating these two solutions. The rest of the parameters have identical solutions. However, these two solutions lead to very different fits for $\left(P+\Delta_{3}\right) / T$ from the $\mathrm{A}_{C P}^{\mathrm{dir}}$ data and hence we present both the solutions.

Since the phases coming from final state interactions should be interpreted as being generated by rescattering due to the presence of resonances, the phases should follow a distinct pattern determined by the masses of the resonances corresponding to particular isospin quantum numbers. The spectrum of the masses of these scalar

TABLE III. The fit value of the parameters and their correlations. (top) Fit values of the parameters. $T, C, \Delta, \kappa^{(\prime)}$, and $K^{(\prime)}$ are in units of $\mathrm{GeV}^{3}$. The angle $\phi$ and the phases $\delta_{0}, \delta_{0}^{\prime}, \delta_{\frac{1}{2}}$, and $\delta_{1}$ are in radians. The parameter $\epsilon_{\delta}$ is dimensionless. The phases $\delta_{i}$ have two solutions, positive and negative. The negative solution is better motivated as explained in the text. The solution for $\left(P+\Delta_{3}\right) / T$ changes accordingly while the solutions for all other parameters remain the same. (bottom) Correlation matrix of the parameters, $\lambda$ being the Wolfenstein parameter in the CKM matrix.

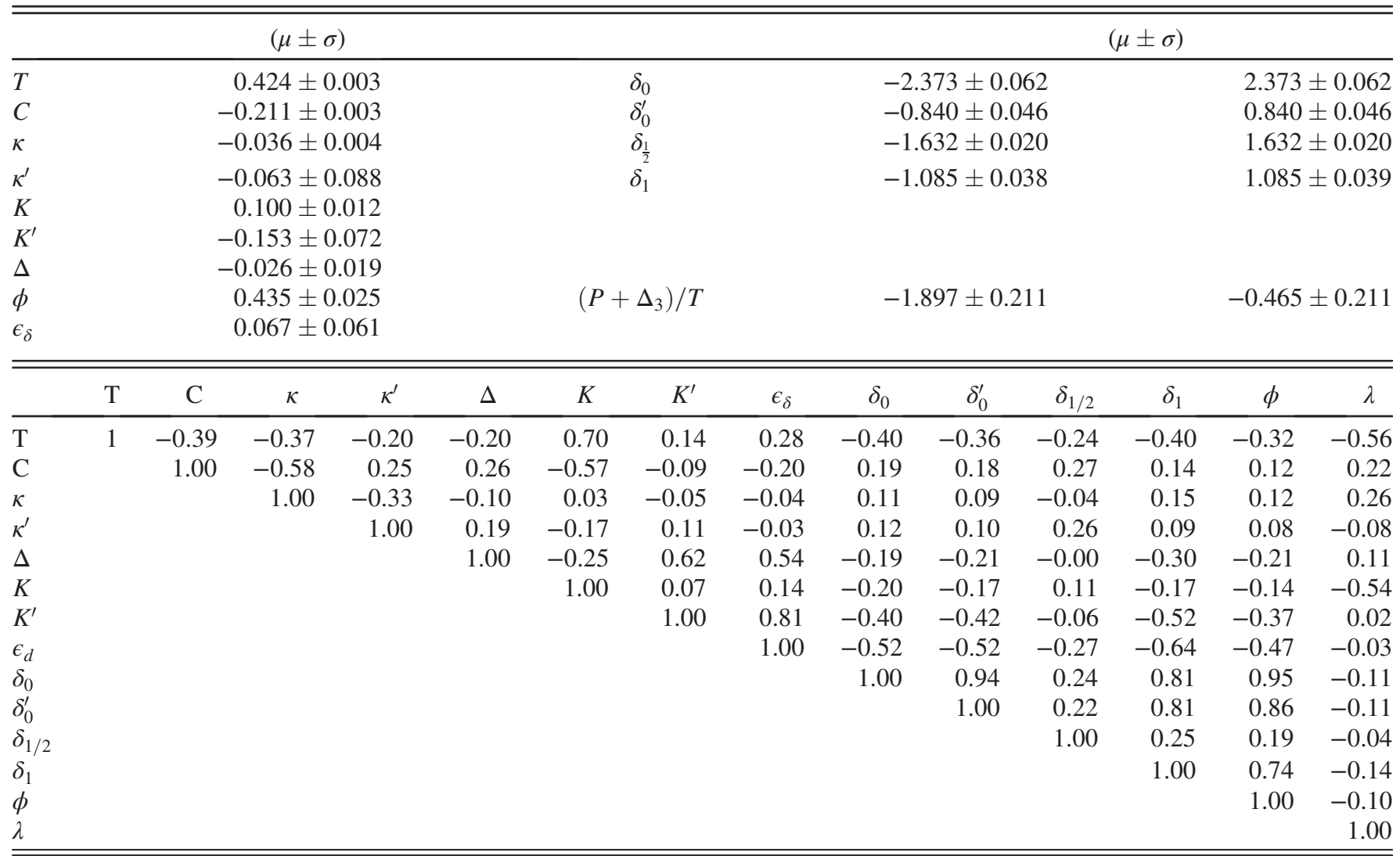



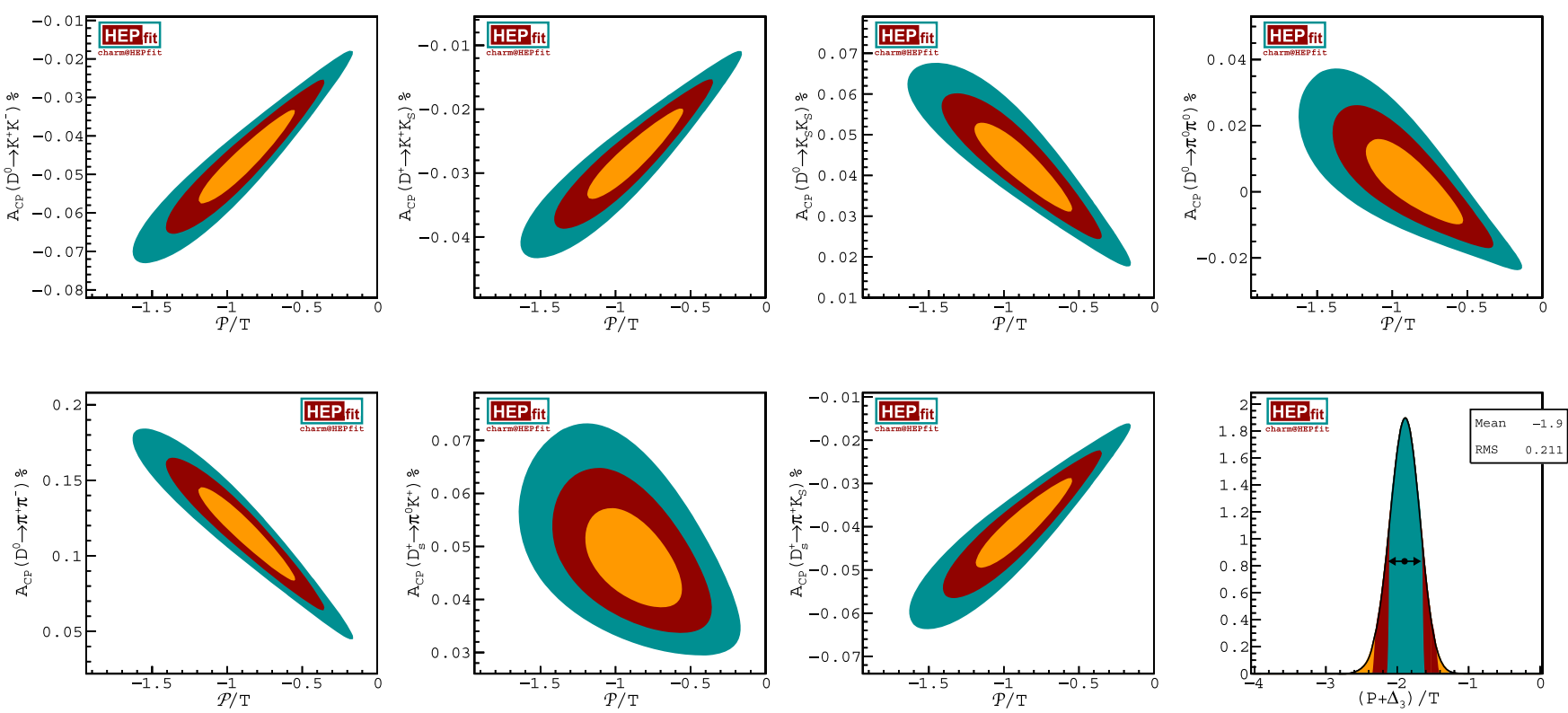

FIG. 1. The correlations between $\mathcal{P} / T$ and the $C P$ asymmetries (given in $\%$ ). HFLAV world average of $\Delta \mathrm{A}_{C P}$ has been used for the fit and these $C P$ asymmetries correspond to the negative solution for the phases. The orange, red, and green regions are the $68 \%, 95 \%$, and 99\% probability regions, respectively. The bottom right-most panel shows the fit to $\left(P+\Delta_{3}\right) / T=\mathcal{P} / T-1$. The green, red, and orange regions are the $68 \%, 95 \%$, and $99 \%$ probability regions, respectively.

resonances can be inferred upon by using the Gell-MannOkubo mass formula [121-124]. From the latter it can be seen that the mass of the resonance corresponding to the $I=1$ channel is smaller than the mass of the resonance corresponding to the $I=1 / 2$ channel. This implies that the strong phase shift due to resonance rescattering in the $I=1$ channel should be larger than that in the $I=1 / 2$ channel. As a consequence, the solution with negative phases seem to be the favorable solution. In the following discussion we shall focus more on the results with the negative solution keeping in mind that it is better motivated.

The strongest constraint, by far, on $\left(P+\Delta_{3}\right) / T$ comes from $\Delta \mathrm{A}_{C P}^{\mathrm{dir}}$. In Fig. 1 we show the posterior distribution of $\left(P+\Delta_{3}\right) / T$. It was pointed out earlier that the $C P$ asymmetries in the different channels are parametrically correlated. Hence the constraints from $\Delta \mathrm{A}_{C P}^{\mathrm{dir}}$ also put constraints on $C P$ asymmetries in the other decay modes. We use this to make predictions for the $C P$ asymmetries in the other SCS channels which we present in Table IV including the single channels $\mathrm{A}_{C P}\left(D^{0} \rightarrow \pi^{+} \pi^{-}\right)$and $\mathrm{A}_{C P}\left(D^{0} \rightarrow K^{+} K^{-}\right)$. The errors in the prediction of the asymmetries vindicate our deduction that amongst the $C P$ asymmetries $\Delta \mathrm{A}_{\mathrm{CP}}^{\mathrm{dir}}$ puts the strongest constraint on $\left(P+\Delta_{3}\right) / T$ by far.

It is important to note here that the $\left(P+\Delta_{3}\right) / T \sim 2$ from the fit making $\left(P+\Delta_{3}\right) / T$ comparable in size to the tree amplitudes parametrized by $T$. This implies that the penguin amplitudes, which appear in the terms proportional to $\left(P+T+\Delta_{3}\right)$, are the same size as the tree amplitudes.
As a result, the penguin amplitudes can no longer be considered the dominant contribution in $C P$ asymmetries of these channels. While it is still sizable compared to the contribution proportional to $T+C$, it can only bring about a factor of few, and not an order of magnitude, enhancement contrary to what was previously expected. This can be clearly gauged from Fig. 1. The recent measurement of $\Delta \mathrm{A}_{C P}$ which is over $5 \sigma$ in significance, also implies that all the asymmetries listed in Table IV are nonzero with a significance of greater than $5 \sigma$ with the important exception of $\mathrm{A}_{C P}\left(D^{0} \rightarrow \pi^{0} \pi^{0}\right)$ that can still be 0 for the negative solution of the phases but not for the positive solution. This parametric correlation between $\Delta \mathrm{A}_{C P}$ and the other $C P$ asymmetries also implies that the predictions of $C P$ asymmetries in these SCS modes using only the $\Delta \mathrm{A}_{C P}$ data has error bars that are much smaller than what is projected as the sensitivity at Belle II with $50 \mathrm{ab}^{-1}$ of data as shown in Table VI. While on one hand this means that many of the $C P$ asymmetries might be beyond the reach of the Belle II experiment, on the other hand it also means that if a $C P$ asymmetry is measured in any of these channels that are far larger than what is predicted here, it will imply a possible necessity for sources of larger $\mathrm{SU}(3)_{F}$ beyond just large phases from FSI. An important test of this will be the measurement of $\mathrm{A}_{C P}\left(D^{0} \rightarrow K_{S} K_{S}\right)$ since its size is quite sensitive to how $\mathrm{SU}(3)_{F}$ is broken.

Finally, we make some predictions from our fit. The branching fraction of the decay mode $D_{s}^{+} \rightarrow K^{+} K_{L}$ is yet unmeasured. However, the sum of the branching fractions for $D_{s}^{+} \rightarrow K^{+} K_{S}$ and $D_{s}^{+} \rightarrow K^{+} K_{L}$ has been measured by Belle yielding [120] 
TABLE IV. Predictions of $C P$ asymmetries using the branching fraction data and the HFLAV average of $\Delta \mathrm{A}_{C P}^{\text {dir }}$.

\begin{tabular}{|c|c|c|c|c|c|}
\hline \multirow[b]{2}{*}{$\underline{\mathrm{A}_{C P}\left(D^{0}\right)}$} & \multicolumn{2}{|c|}{$(\mu \pm \sigma)(\%)$} & \multirow[b]{2}{*}{$\mathrm{A}_{C P}\left(D_{(s)}^{+}\right)$} & \multicolumn{2}{|c|}{$(\mu \pm \sigma)(\%)$} \\
\hline & $\delta_{i} \rightarrow-\mathrm{ve}$ & $\delta_{i} \rightarrow+\mathrm{ve}$ & & $\delta_{i} \rightarrow-\mathrm{ve}$ & $\delta_{i} \rightarrow+\mathrm{ve}$ \\
\hline$\overline{D^{0} \rightarrow \pi^{+} \pi^{-}}$ & $0.117 \pm 0.020$ & $0.118 \pm 0.020$ & $D^{+} \rightarrow K^{+} K_{S}$ & $-0.028 \pm 0.005$ & $-0.026 \pm 0.005$ \\
\hline$D^{0} \rightarrow \pi^{0} \pi^{0}$ & $0.004 \pm 0.009$ & $0.079 \pm 0.010$ & $D_{S}^{+} \rightarrow \pi^{+} K_{S}$ & $-0.040 \pm 0.007$ & $-0.036 \pm 0.007$ \\
\hline$D^{0} \rightarrow K^{+} K^{-}$ & $-0.047 \pm 0.008$ & $-0.046 \pm 0.008$ & $D_{s}^{+} \rightarrow \pi^{0} K^{+}$ & $0.048 \pm 0.006$ & $-0.003 \pm 0.004$ \\
\hline$D^{0} \rightarrow K_{S} K_{S}$ & $0.043 \pm 0.007$ & $0.038 \pm 0.007$ & & & \\
\hline
\end{tabular}

$$
\begin{aligned}
& \mathrm{BR}\left(D_{s}^{+} \rightarrow K^{+} K_{S}\right)+\mathrm{BR}\left(D_{s}^{+} \rightarrow K^{+} K_{L}\right) \\
& \quad=(29.5 \pm 1.1 \pm 0.9) \times 10^{-3}
\end{aligned}
$$

while the branching fraction $\operatorname{BR}\left(D_{s}^{+} \rightarrow K^{+} K_{S}\right)=$ $(15.0 \pm 0.5) \times 10^{-3}$. Several predictions have been made in the past for the rate asymmetry between $D_{s}^{+} \rightarrow K^{+} K_{L}$ and $D_{s}^{+} \rightarrow K^{+} K_{S}$ which is tantamount to predicting the branching fraction of the former since the branching fraction of the latter mode is measured to a very good precision. In [81,125] the branching fraction of $D_{S}^{+} \rightarrow$ $K^{+} K_{L}$ is predicted to be smaller than the branching fraction of $D_{s}^{+} \rightarrow K^{+} K_{S}$. In contrast, we predict

$$
\operatorname{BR}\left(D_{s}^{+} \rightarrow K^{+} K_{L}\right)=(14.98 \pm 0.39) \times 10^{-3},
$$

which is almost equal to the branching fraction of $D_{s}^{+} \rightarrow$ $K^{+} K_{S}$ with the central value of the former being greater than the latter. The discrepancy is discussed in the next section where we also discuss the rate asymmetry and compare with results in the literature. If we do not use the result in Eq. (22) we get

$$
\operatorname{BR}\left(D_{s}^{+} \rightarrow K^{+} K_{L}\right)=(15.01 \pm 0.47) \times 10^{-3} .
$$

We also predict the relative strong phase between the amplitudes of the modes $D^{0} \rightarrow K^{+} \pi^{-}$and $D^{0} \rightarrow K^{-} \pi^{+}$. The world average of the measured value of this phase is $\left(9.3_{-9.2}^{+8.3}\right)^{\circ}[102,126]$ when one assumes that there is no $C P$ violation in the DCS decays. From our fit we get [127]

$$
\delta_{K \pi}=\delta_{K^{-} \pi^{+}}-\delta_{K^{+} \pi^{-}}=3.14^{\circ} \pm 5.69^{\circ}
$$

which is compatible with the measured value. Various other estimates of $\delta_{K \pi}$ can be found in [79,128-131]. In the exact $\mathrm{SU}(3)_{F}$ limit $\delta_{K \pi}$ should be 0 [132-134], the deviation from which, as indicated by the fit result, underscores the significance of $\mathrm{SU}(3)_{F}$ breaking through strong phases in the framework that we use.

\section{B. Rate asymmetries}

One can also define rate asymmetries involving interference of CA and DCS decays of the neutral $D^{0}$ meson to the neutral $K \pi$ final state. A method for measuring this was first proposed in [135]. The rate asymmetry for the neutral $D^{0}$ initial state is defined as

$$
R\left(D^{0}, \pi^{0}\right) \equiv \frac{\Gamma\left(D^{0} \rightarrow K_{S} \pi^{0}\right)-\Gamma\left(D^{0} \rightarrow K_{L} \pi^{0}\right)}{\Gamma\left(D^{0} \rightarrow K_{S} \pi^{0}\right)+\Gamma\left(D^{0} \rightarrow K_{L} \pi^{0}\right)}
$$

For the charged $D^{+}$in the initial state, the rate asymmetry is defined as $R\left(D^{+}, \pi^{+}\right)$with the substitutions $D^{0} \rightarrow D^{+}$and $\pi^{0} \rightarrow \pi^{+}$. For $D_{s}^{+}$the rate asymmetry is defined as $R\left(D_{s}^{+}, K^{+}\right)$with the substitution $D^{0} \rightarrow D_{s}^{+}$and $\pi^{0} \rightarrow$ $K^{+}$in the above relation. The rate asymmetry in
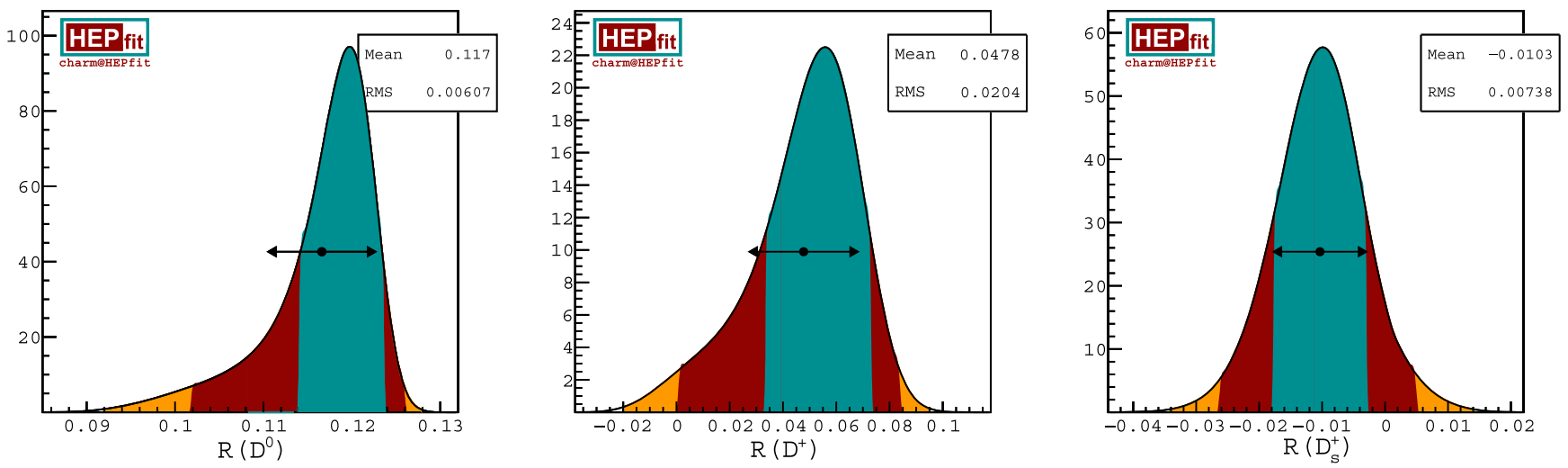

FIG. 2. Fit results for $R\left(D^{0}, \pi^{0}\right), R\left(D^{+}, \pi^{+}\right)$, and $R\left(D_{s}^{+}, K^{+}\right)$using the branching fraction data in Table II and the HFLAV world average of $\Delta \mathrm{A}_{C P}$. The green, red, and orange regions are the $68 \%, 95 \%$, and $99 \%$ probability regions respectively. 
TABLE V. Estimates of $R\left(D^{0}, \pi^{0}\right), R\left(D^{+}, \pi^{+}\right)$, and $R\left(D_{s}^{+}, K^{+}\right)$from the existing literature.

\begin{tabular}{|c|c|c|c|c|c|}
\hline$R\left(D^{0}, \pi^{0}\right)$ & & $R\left(D^{+}, \pi^{+}\right)$ & & $R\left(D_{s}^{+}, K^{+}\right)$ & \\
\hline$\sim 10 \%$ & [135] & $-0.010 \pm 0.026$ & [131] & $-0.003_{-0.017}^{+0.019}$ & [139] \\
\hline$\sim 0.106$ & [131] & $-0.006_{-0.028}^{+0.033}$ & [139] & $-0.0022 \pm 0.0087$ & [92] \\
\hline 0.107 & {$[92]$} & $-0.005 \pm 0.013$ & {$[92]$} & $-0.008 \pm 0.007$ & {$[101,140]$} \\
\hline $0.09_{-0.02}^{+0.04}$ & {$[81]$} & $-0.019 \pm 0.016$ & [101] & $0.11_{-0.14}^{+0.04}$ & [81] \\
\hline $0.113 \pm 0.001$ & [125] & $0.025 \pm 0.008$ & [125] & $0.012 \pm 0.006$ & {$[125]$} \\
\hline
\end{tabular}

Eq. (26) leads us to another U-spin breaking parameter $\epsilon_{0}^{\prime}$, the real part of which is can be shown to be given by

$$
\operatorname{Re}\left(\epsilon_{0}^{\prime}\right)=\frac{R\left(D^{0}, \pi^{0}\right)}{4 \tan ^{2} \theta_{C}}-\frac{1}{2}
$$

From a CLEO Collaboration measurement of $K_{S}-K_{L}$ asymmetry [136], the measured values for $R\left(D^{0}, \pi^{0}\right)$, $R\left(D^{+}, \pi^{+}\right)$are

$$
\begin{aligned}
R\left(D^{0}, \pi^{0}\right)^{\mathrm{CLEO}} & =0.108 \pm 0.025 \pm 0.024, \\
R\left(D^{+}, \pi^{+}\right)^{\mathrm{CLEO}} & =0.022 \pm 0.016 \pm 0.018,
\end{aligned}
$$

leading to a value of $\operatorname{Re}\left(\epsilon_{0}^{\prime}\right)=0.00 \pm 0.16$ [137]. We compute $R\left(D^{0}, \pi^{0}\right), R\left(D^{+}, \pi^{+}\right)$and $\operatorname{Re}\left(\epsilon_{0}^{\prime}\right)$ from our fit to the branching fractions and $C P$ asymmetries and get (cf. Fig. 2) [138]

$$
\begin{aligned}
R\left(D^{0}, \pi^{0}\right) & =0.1166 \pm 0.0061, \\
R\left(D^{+}, \pi^{+}\right) & =0.048 \pm 0.020 \\
\operatorname{Re}\left(\epsilon_{0}^{\prime}\right) & =0.045 \pm 0.029
\end{aligned}
$$

which is in fair agreement with the CLEO measurements. The various predictions for $R\left(D^{0}, \pi^{0}\right)$ and $R\left(D^{+}, \pi^{+}\right)$that have been made previously are listed in Table V.

We present a prediction of $R\left(D_{s}^{+}, K^{+}\right)$(cf. Fig. 2) [141]:

$$
R\left(D_{s}^{+}, K^{+}\right)=-0.0103 \pm 0.0074,
$$

which can be compared with other predictions made in the past as listed in Table V. All the results are reasonably compatible. However, these imply that $\operatorname{BR}\left(D_{s}^{+} \rightarrow\right.$ $\left.K^{+} K_{S}\right)>\operatorname{BR}\left(D_{s}^{+} \rightarrow K^{+} K_{L}\right)$ in $[81,125]$, whereas for the rest the contrary is true if one considers the central values of the ratio. Since this rate asymmetry depends on the estimate of the strong phases, a measurement of the latter can be used to test our predictions of the strong phases.

\section{AMPLITUDE RELATIONS AND SU $(3)_{F}$ BREAKING}

While our parametrization is well motivated by $\mathrm{SU}(3)_{F}$ arguments, it is also good to check if there are some ways of validating it. Here we follow a more general theoretical construction of $\mathrm{SU}(3)_{F}$ arguments put forward by Gronau in [137] which also allows for a measure of the degree at which $\mathrm{SU}(3)_{F}$ is broken by applying a higher order perturbation expansion in $\mathrm{SU}(3)_{F}$ breaking. The amplitude relations for $D^{0}$ decays to pairs of neutral pseudoscalar mesons can be written as

$$
\begin{aligned}
& R_{1} \equiv \frac{\left|A\left(D^{0} \rightarrow K^{+} \pi^{-}\right)\right|}{\left|A\left(D^{0} \rightarrow \pi^{+} K^{-}\right)\right| \tan ^{2} \theta_{C}}, \quad R_{2} \equiv \frac{\left|A\left(D^{0} \rightarrow K^{+} K^{-}\right)\right|}{\left|A\left(D^{0} \rightarrow \pi^{+} \pi^{-}\right)\right|}, \\
& R_{3} \equiv \frac{\left|A\left(D^{0} \rightarrow K^{+} K^{-}\right)\right|+\left|A\left(D^{0} \rightarrow \pi^{+} \pi^{-}\right)\right|}{\left|A\left(D^{0} \rightarrow \pi^{+} K^{-}\right)\right| \tan \theta_{C}+\left|A\left(D^{0} \rightarrow K^{+} \pi^{-}\right)\right| \tan ^{-1} \theta_{C}}, \\
& R_{4} \equiv \sqrt{\frac{\left|A\left(D^{0} \rightarrow K^{+} K^{-}\right)\right|\left|A\left(D^{0} \rightarrow \pi^{+} \pi^{-}\right)\right|}{\left|A\left(D^{0} \rightarrow \pi^{+} K^{-}\right)\right|\left|A\left(D^{0} \rightarrow K^{+} \pi^{-}\right)\right|}}
\end{aligned}
$$

These four ratios are not mutually independent. They obey a trivial identity

$$
R_{4}=R_{3} \sqrt{\frac{1-\left[\left(R_{2}-1\right) /\left(R_{2}+1\right)\right]^{2}}{1-\left[\left(R_{1}-1\right) /\left(R_{1}+1\right)^{2}\right.}}
$$

It can be shown that $R_{i}=1$ in the limit of $\mathrm{SU}(3)_{F}$ and the relation

$$
\begin{aligned}
\Delta R \equiv & R_{3}-R_{4} \\
& +\frac{1}{8}\left[\left(\sqrt{2 R_{1}-1}-1\right)^{2}-\left(\sqrt{2 R_{2}-1}-1\right)^{2}\right] \\
= & \mathcal{O}\left(\epsilon_{1}^{4}, \epsilon_{2}^{4}\right)+\mathcal{O}\left(\hat{\delta}_{1} \epsilon_{1}^{2}, \hat{\delta}_{2} \epsilon_{2}^{2}\right)
\end{aligned}
$$

differs from zero by terms of the order $\mathcal{O}\left(\epsilon_{1}^{4}, \epsilon_{2}^{4}\right)+$ $\mathcal{O}\left(\hat{\delta}_{1} \epsilon_{1}^{2}, \hat{\delta}_{2} \epsilon_{2}^{2}\right)$, where $\epsilon_{i}$ and $\hat{\delta}_{i}$ are $\mathrm{U}$-spin and Isospin breaking terms, respectively. One can then write the real parts of the $\mathrm{SU}(3)_{F}$ breaking parameters $\epsilon_{1}$ and $\epsilon_{2}$ as

$$
\begin{aligned}
\operatorname{Re}\left(\epsilon_{i}\right)= & \frac{1}{2}\left(\sqrt{2 R_{i}-1}-1\right)-\operatorname{Re}\left(\hat{\delta}_{i}\right)-2 \operatorname{Re}\left(\hat{\delta}_{i}\right) \operatorname{Re}\left(\epsilon_{i}\right) \\
& +\mathcal{O}\left(\hat{\delta}_{i} \epsilon_{i}\right)+\mathcal{O}\left(\epsilon_{i}^{3}\right)
\end{aligned}
$$

with $i=1,2$. The U-spin breaking in $D^{0} \rightarrow K^{+} \pi^{-}$is denoted by $\epsilon_{1}$ and that in $D^{0} \rightarrow K^{+} K^{-}$is denoted by $\epsilon_{2}$. It 

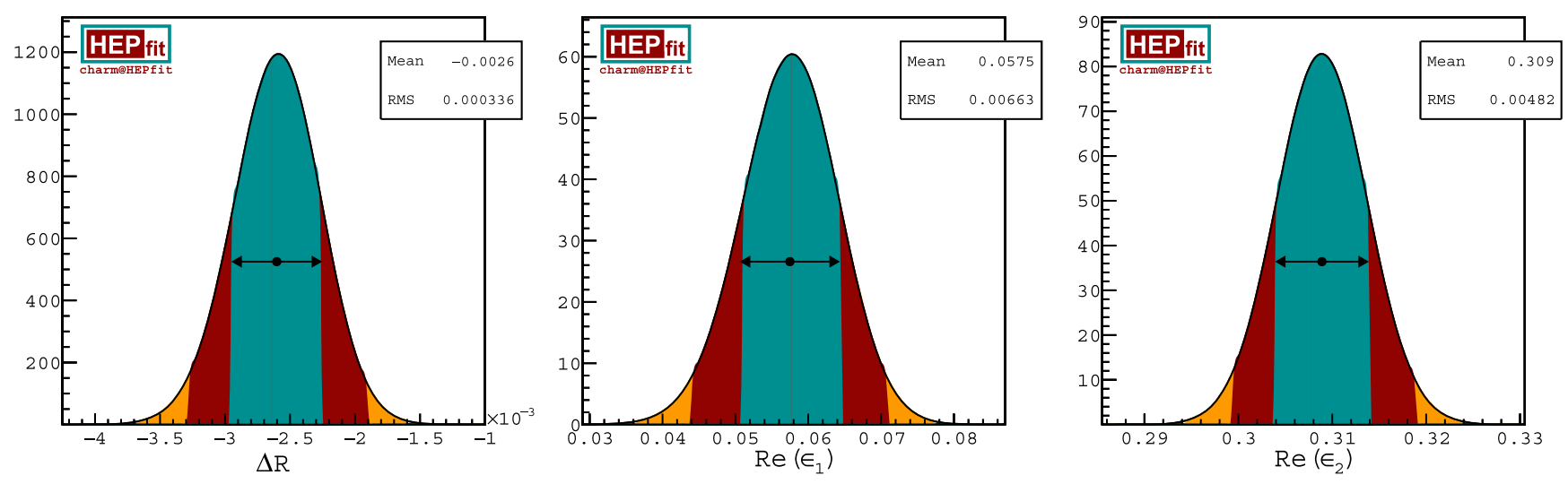

FIG. 3. Fit results for $\Delta R, \epsilon_{1}$, and $\epsilon_{2}$ using the branching fraction data in Table II and the HFLAV world average of $\Delta \mathrm{A}_{C P}$. The green, red, and orange regions are the $68 \%, 95 \%$, and $99 \%$ probability regions respectively.

is expected [137] that $\epsilon_{2}$ quantifies breaking in both the tree and penguin amplitudes while $\epsilon_{1}$ quantifies the breaking in only tree amplitudes. Hence, the former is expected to be somewhat larger than the latter. In our work we do not consider isospin breaking and hence $\hat{\delta}_{i}=0$. We test these relations in our paramterization of the amplitudes and use the parameters extracted from the branching fractions as inputs. We find a fair agreement with the results quoted in [137] for $\Delta R, \operatorname{Re}\left(\epsilon_{1}\right)$ and $\operatorname{Re}\left(\epsilon_{2}\right)$ as is evident from Fig. 3.

\section{Correlations between $\boldsymbol{C P}$ asymmetries}

As a second test of our parametrization we propose the correlation between the $C P$ asymmetries that we have earlier explained are parametrically correlated. Since the asymmetries are correlated through the combination of parameters, $\left(P+\Delta_{3}\right) / T$, it is possible to combine the expression for the asymmetries to obtain relations between them. By considering the $\pi \pi$ and $K K$ final states we have, symbolically,

$$
\begin{aligned}
\mathrm{A}_{C P}(D \rightarrow K K)= & f_{K K}(\vec{p}) A_{C P}\left(D^{0} \rightarrow \pi^{+} \pi^{-}\right) \\
& +g_{K K}(\vec{p}) A_{C P}\left(D^{0} \rightarrow \pi^{0} \pi^{0}\right)+h_{K K}(\vec{p}),
\end{aligned}
$$

where $f_{K K}(\vec{p}), g_{K K}(\vec{p})$ and $h_{K K}(\vec{p})$ are functions of $\vec{p}=$ $\left\{T, C, \kappa, \kappa^{\prime}, K, K^{\prime}, \Delta, \phi, \epsilon_{\delta}, \delta_{0}, \delta_{0}^{\prime}, \delta_{\frac{1}{2}}, \delta_{1}\right\}$ and depend on the final $K K$ pair. With the central values for the parameters from our fits in Table III we get for negative phases

$$
\begin{aligned}
\mathrm{A}_{C P}\left(D^{0} \rightarrow K^{+} K^{-}\right) & =-0.657 A_{C P}\left(D^{0} \rightarrow \pi^{+} \pi^{-}\right)+0.750 A_{C P}\left(D^{0} \rightarrow \pi^{0} \pi^{0}\right)+2.78 \times 10^{-4}, \\
\mathrm{~A}_{C P}\left(D^{0} \rightarrow K_{S} K_{S}\right) & =3.47 A_{C P}\left(D^{0} \rightarrow \pi^{+} \pi^{-}\right)-8.88 A_{C P}\left(D^{0} \rightarrow \pi^{0} \pi^{0}\right)-3.28 \times 10^{-3} .
\end{aligned}
$$

When we consider the fit with positive phases the constant terms change their signs. In the case in which we consider the limit $\Delta_{4} \rightarrow 0$ we have

$$
\begin{aligned}
\mathrm{A}_{C P}\left(D^{0} \rightarrow K^{+} K^{-}\right) & =-0.394 \mathrm{~A}_{C P}\left(D^{0} \rightarrow \pi^{+} \pi^{-}\right)-1.05 \times 10^{-6} \\
\mathrm{~A}_{C P}\left(D^{0} \rightarrow K_{S} K_{S}\right) & =0.342 \mathrm{~A}_{C P}\left(D^{0} \rightarrow \pi^{+} \pi^{-}\right)+2.75 \times 10^{-5} \\
\mathrm{~A}_{C P}\left(D^{0} \rightarrow \pi^{0} \pi^{0}\right) & =0.352 \mathrm{~A}_{C P}\left(D^{0} \rightarrow \pi^{+} \pi^{-}\right)-3.72 \times 10^{-4}
\end{aligned}
$$

Likewise, the $C P$ asymmetries in the other channels can also be correlated. These correlations between the predicted asymmetries are plotted in Fig. 4 and includes several SCS decay modes in which $C P$ violation is possible. A deviation from these correlations would indicate a breakdown of our parametrization. The correlations have been derived by using only the branching fraction data and the measurement of $\Delta \mathrm{A}_{C P}$ from $\mathrm{LHCb}$. Most notably, the formalism we use renders the $C P$ asymmetry in $D^{0} \rightarrow K_{S} K_{S}$ completely correlated to $\Delta \mathrm{A}_{C P}$ since the weak exchange diagram present in the $\Delta U=0$ part of the amplitude of the former decay mode, and absent in the latter, is generated by rescattering and is not an independent contribution. 

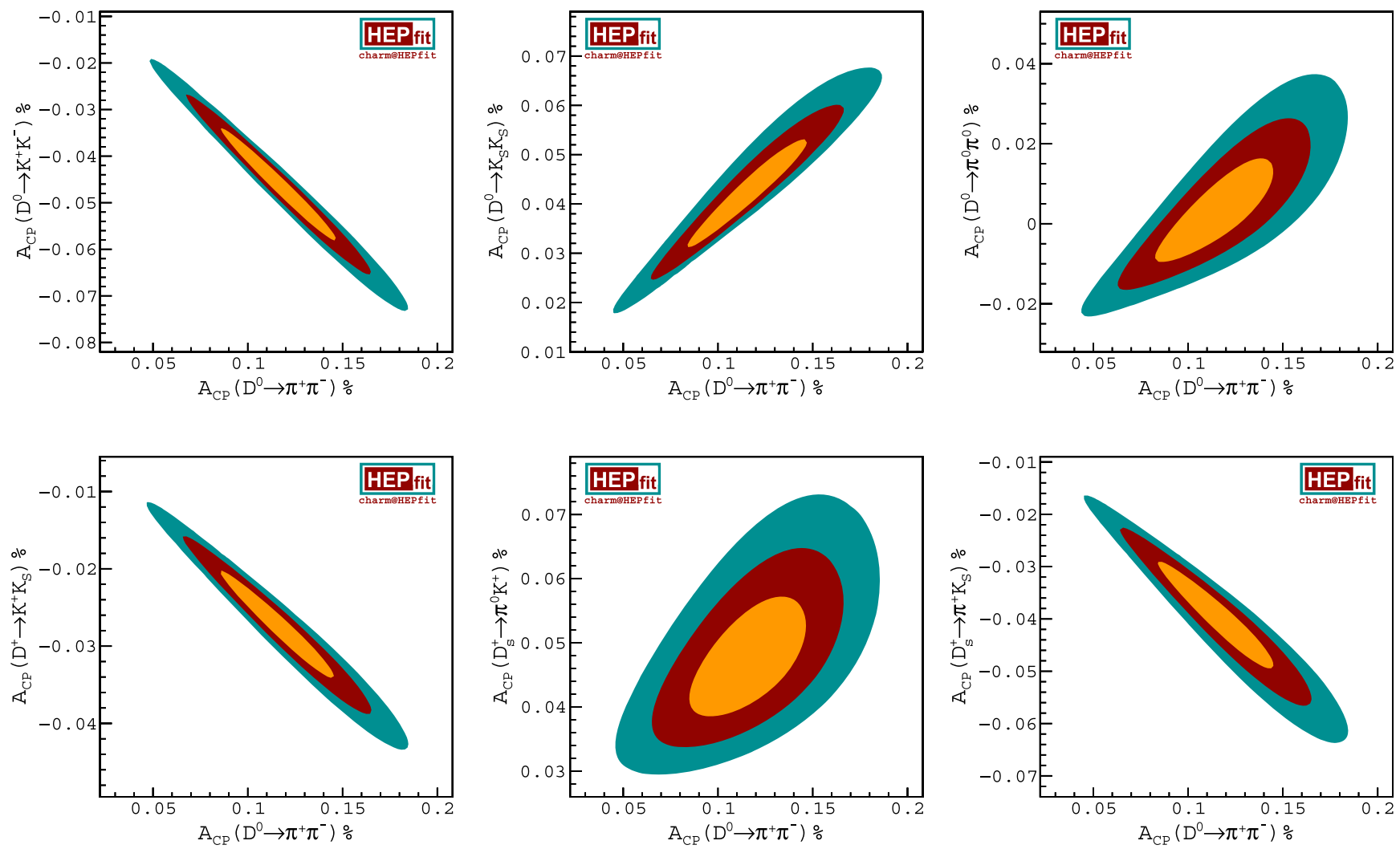

FIG. 4. Correlations between asymmetries (in \%) as given in Eq. (37) using the branching fraction data in Table II and the HFLAV world average of $\Delta \mathrm{A}_{C P}$ quoted in Sec. III. The orange, red, and green regions are the $68 \%$, 95\%, and $99 \%$ probability regions, respectively.

\section{E. Constraints on penguin amplitudes from future measurements}

With Belle II starting up and LHCb having built a very strong charm program over the past few years, it is instructive to see what these measurements will mean in terms of constraining the penguin amplitudes. While the measurements of the branching fractions are expected to improve significantly too, this will not additionally constrain the penguin amplitudes directly. However, the ratio $\left(P+\Delta_{3}\right) / T$ would certainly benefit from an improved determination of $T$. Considering $T$ is already extracted at a precision of less than $O(1 \%)$, improvements in this parameter will leave a negligible effect. On the other hand, not all the phases appearing in the SCS decays are very well constrained. An improvement in these would certainly improve the constraints on the penguin amplitudes. In particular, an improved measurement of $D^{0} \rightarrow K_{S} K_{S}$, which is nonvanishing only when $\mathrm{SU}(3)_{F}$ is broken, is

TABLE VI. Numbers used to generate the constraints on $\mathcal{P} / T$ from future experiments. The column marked "Current fit" shows the rms from our prediction of the asymmetries using the branching fraction data and the LHCb measurement of $\Delta \mathrm{A}_{C P}$ only and for the negative solution for the phases.

\begin{tabular}{|c|c|c|c|c|}
\hline \multirow[b]{2}{*}{$\mathrm{A}_{C P}$ (channel) } & \multirow[b]{2}{*}{ Mode $(\%)$} & \multicolumn{3}{|c|}{$\operatorname{rms}(\%)$} \\
\hline & & Current fit & Belle II $50 \mathrm{ab}^{-1}$ [142] & $\mathrm{LHCb} 50 \mathrm{fb}^{-1}$ [143] \\
\hline$D^{0} \rightarrow \pi^{+} \pi^{-}$ & 0.1174 & 0.020 & 0.05 & $\cdots$ \\
\hline$D^{0} \rightarrow \pi^{0} \pi^{0}$ & -0.0034 & 0.009 & 0.09 & $\ldots$ \\
\hline$D^{0} \rightarrow K^{+} K^{-}$ & -0.0465 & 0.008 & 0.03 & $\ldots$ \\
\hline$D^{0} \rightarrow K_{S} K_{S}$ & 0.0431 & 0.007 & 0.17 & $\ldots$ \\
\hline$D^{+} \rightarrow \stackrel{K^{+}}{K_{S}}$ & -0.0276 & 0.005 & 0.05 & $\ldots$ \\
\hline$D_{s}^{+} \rightarrow \pi^{+} K_{S}$ & -0.0403 & 0.007 & 0.29 & $\ldots$ \\
\hline$\Delta \mathrm{A}_{C P}$ & -0.164 & $\ldots$ & $\ldots$ & 0.01 \\
\hline
\end{tabular}




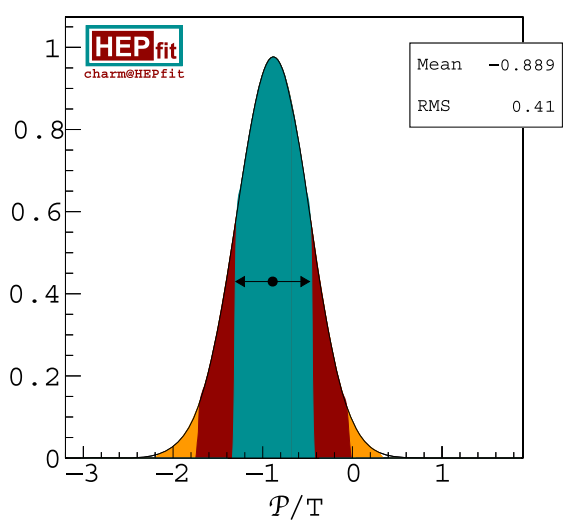

(a) BII-50

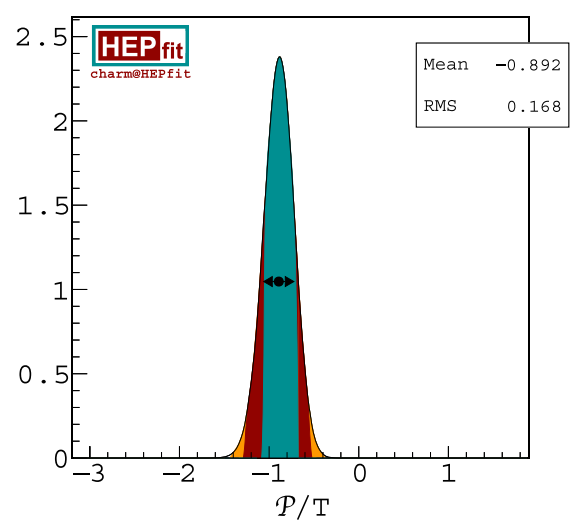

(b) $\mathrm{BII}-50+\Delta \mathrm{A}_{\mathrm{CP}}$

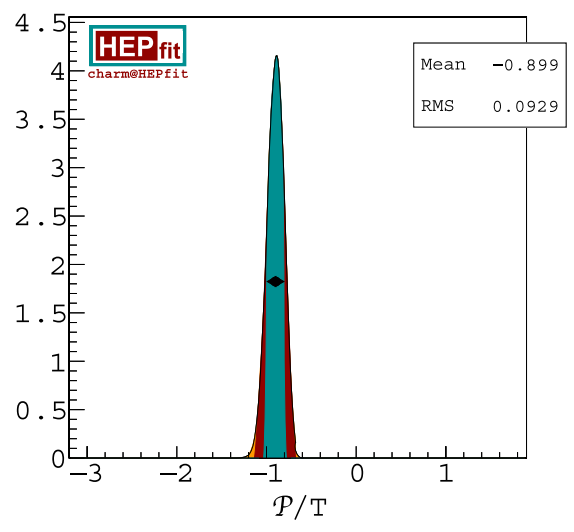

(c) $\mathrm{BII}-50+\mathrm{LHCb}-50$

FIG. 5. Fit results for $\mathcal{P} / T$ using the branching fraction data in Table II and $C P$ asymmetries listed in Table VI. The green, red, and orange regions are the $68 \%, 95 \%$, and $99 \%$ probability regions, respectively. (BII-50: Belle II $50 \mathrm{ab}^{-1}$; $\mathrm{LHCb}^{-50:} \mathrm{LHCb}_{50} \mathrm{fb}^{-1}$.)

quite important for further constraining the parameters that arise from this breaking specially because the branching ratio of this channel is not well measured currently.

To keep the analysis simple and on the more conservative side we do not take into account any improvement in the measurement of the branching fractions. We project the central values of the $C P$ asymmetries using their value at the global mode of the current fit and use the errors projected by the experiments. We use projections for Belle II at $50 \mathrm{ab}^{-1}$ for various asymmetries. We also use the projected measurement of $\Delta \mathrm{A}_{C P}$ at $\mathrm{LHCb} 50 \mathrm{fb}^{-1}$ data. Finally, we combine all these projected measurements. These are tabulated in Table VI.

In Fig. 5 we show how the constraints on $\mathcal{P} / T$ will change with additional data. As is evident, the constraints are not much better than what we see in Fig. 1 with only the full Belle II data. The reason for this is that the projected precision of measurement of these asymmetries from the full Belle II data of $50 \mathrm{fb}^{-1}$ is comparable or worse than the precision of the prediction of the asymmetries from the current measurement of $\Delta \mathrm{A}_{C P}$ as can be seen from Table VI. Once the measurement of $\Delta \mathrm{A}_{C P}$ improves, the constraint on $\mathcal{P} / T$ gets much better, but significantly so only after $50 \mathrm{fb}^{-1}$ of data from LHCb.

\section{SUMMARY}

The main purpose of this work is to take advantage of the high precision reached by the measurements of the branching ratios in two particle final states consisting of kaons and (or) pions of the pseudoscalar charmed particles to deduce the predictions of the Standard Model for the $C P$ violating asymmetries in their decays. To this extent we have constructed amplitudes in agreement with the measured branching ratios, where the $\mathrm{SU}(3)_{F}$ violations come mainly from the final state interaction and from the nonconservation of the strangeness changing vector currents.

So in this work we extend the formalism presented in [91] with a larger menu of branching fractions for $D \rightarrow P P$ with $P=K, \pi$ but excluding the branching fractions which have $\eta / \eta^{\prime}$ in the final state. We extend the old parametrization with the parameters $K^{(\prime)}$ and $\kappa^{(\prime)}$ to address $\mathrm{SU}(3)_{F}$ breaking effects both in the tree and colour suppressed amplitudes. We introduce $\epsilon_{\delta}$ to address the splitting of the phases due to mass splitting between the $D^{0,+}$ and the $D_{s}^{+}$. Another parameter $\Delta$ is included to address the decays of $D_{(s)}^{+}$mesons. To accommodate for $C P$ asymmetry in the SCS decays we introduce three parameters $P, \Delta_{3}$, and $\Delta_{4}$. The latter is parametrically suppressed due to an approximate selection rule. The former two cannot be resolved from $C P$ asymmetries of the SCS decays we consider and hence only the sum can be extracted from data and we deem its ratio with $T$ as the parameter relevant for the fit.

We perform a fit of the parameters to the branching fractions and $\Delta \mathrm{A}_{C P}$ using HEPFIT and predict several $C P$ asymmetries using our parametrization. In our framework, ignoring very small effects, the $C P$ asymmetries show distinct correlations which can serve as a test of our framework. We also explore $\mathrm{SU}(3)_{F}$ breaking effects as advocated by Gronau [137] and find a good agreement with the results from that work. The rate asymmetries extracted from the branching fraction data agrees well with the CLEO collaboration data. As a future extension of this work, we will extend the parametrization to final states with $\eta / \eta^{\prime}$.

Within the ambit of our work we find reasonable success in trying to parametrize $D \rightarrow P P$ decays within a $\mathrm{SU}(3)_{F}$ framework. The important conclusions of our work are the following:

(i) We succeed in describing the measured branching fractions by invoking $\mathrm{SU}(3)_{F}$ breaking using large 
phases from FSI, nonconservation of the strangeness changing vector current and slight shifts in the reduced matrix elements for CA and the DCS vs the SCS decay amplitudes. This does not require the introduction of the parameter $P, \Delta_{3}$ or $\Delta_{4}$.

(ii) The values of the FSI phases, when considering the negative solutions, fall nicely along the pattern of the expected mass ordering of the resonance from the presence of which these FSI phases are generated. This also fixes the imaginary parts, which are relevant for the $C P$ violating asymmetries. The negative solution is motivated by considering the masses of the resonances to be arranged according to the Gell-Man-Ne'emanOkubo mass formula which requires the strong phase in the $I=1$ channel to be larger than that in the $I=1 / 2$ channel.

(iii) Once we relate the 15 in the $\Delta U=0$ part of the amplitude to that in the $\Delta U=1$ part of the amplitude the asymmetries depend on three new parameters. Of these, the combination $P+T+\Delta_{3}$ incorporates the uncertain strength of the penguin contributions. Then we apply an approximate selection rule that forbids the simultaneous creation of a $d \bar{d}$ and a $s \bar{s}$ pair, similar to the OZI rule, to the penguin annihilation contribution coming from the 3 . Hence, the third parameter, $\Delta_{4}$, is expected to be small by this approximate selection rule and contributes mainly to the asymmetry in $D^{0} \rightarrow K_{S} K_{S}$ as can be seen from the $\mathrm{SU}(3)_{F}$ limit. Moreover, the terms proportional to $T+C$ are constrained by the branching fraction data. The combination $P+\Delta_{3}$ cannot be disentangled from measurements. Hence all the $C P$ asymmetries depend on this combination of parameter and are thus correlated.

(iv) We show that amongst the current measurement of $C P$ asymmetries, $\Delta \mathrm{A}_{C P}^{\mathrm{dir}}$ is by far the strongest constraint on the combination $\left(P+\Delta_{3}\right) / T$. We use this fact and the parametric correlation between the $\Delta U=0$ part of the amplitudes to predict several asymmetries which are listed in Table IV. Since $\triangle \mathrm{A}_{C P}^{\mathrm{dir}}$ constrains the penguin amplitude to $\mathcal{P} \sim \mathcal{O}(T)$, the part proportional to it no longer dominates the $C P$ asymmetries. Indeed the part proportional to $(T+C)$ becomes sizable in comparison. Hence the penguin amplitudes can no longer be expected to bring about an order of magnitude enhancement beyond the $1 \%$ level in the $C P$ asymmetries of several channel and can enhance them by only a factor of few. This lies in contrast with what was previously expected as the effect of penguin amplitudes in the $C P$ asymmetries of SCS $D \rightarrow P P$ decays. (v) With the correlations between the asymmetries and the direction pointed at by the data we can propose methods for validating our $\mathrm{SU}(3)_{F}$ framework by looking at rate asymmetries between several $K_{S}-K_{L}$ final states and the correlation between $C P$ asymmetries in different channels. In particular, as a consequence of the strong phases determined by the fit, we predict the yet unmeasured rate asymmetry:

$$
R\left(D_{s}^{+}, K^{+}\right)=-0.0103 \pm 0.0074 .
$$

(vi) When we choose the negative solution for the phases, we also predict

$$
\delta_{K \pi}=\delta_{K^{-} \pi^{+}}-\delta_{K^{+} \pi^{-}}=3.14^{\circ} \pm 5.69^{\circ} .
$$

In this framework of $\mathrm{SU}(3)_{F}$ breaking that is driven by large phase from FSI due to rescattering through scalar resonances, it can be shown that $C P$ asymmetries in all SCS modes are constrained to the per mille level by the current measurement of $\Delta \mathrm{A}_{C P}^{\mathrm{dir}}$.

\section{ACKNOWLEDGMENTS}

A. P. would like to acknowledge partial support from ERC Ideas Starting Grant No. 279972 "NPFlavour" while most of this work was being done. We would like to thank Alessandra Pugliese and Maurizio Lusignoli for fruitful discussions during the initial stages of the work. We would like to thank Luca Silvestrini and Enrico Franco for their help and support for several aspects of this work.

\section{APPENDIX: POSTERIOR DISTRIBUTIONS OF THE PARAMETERS FOR THE FULL FIT}

The fit of the parameters to the branching fractions and $\Delta \mathrm{A}_{C P}^{\mathrm{dir}}$ and the predictions for the $C P$ asymmetries was done with HEPFIT. A model was built specifically for this purpose. The code necessary for replicating this analysis can be made available on request. In Fig. 6 we show the posterior distributions of the parameters from the fit with the mean and rms listed in Table II and $\Delta \mathrm{A}_{C P}^{\mathrm{dir}}$ quoted in Sec. III. Only the posteriors for $\delta_{0}$ and $\kappa^{\prime}$ show some deviation from being Gaussian distributions. The two phases $\delta_{0}$ and $\delta_{0}^{\prime}$ and the angle $\phi$ that appear in the SCS decays are highly correlated. We show the correlation plots for these parameters in the bottom three plots of Fig. 6 . The values of the CKM parameters used in these fits are from the UTfit average [144]:

$$
\begin{aligned}
& \lambda=0.22534 \pm 0.00089, \quad A=0.833 \pm 0.012, \\
& \bar{\rho}=0.153 \pm 0.013, \quad \bar{\eta}=0.343 \pm 0.011 .
\end{aligned}
$$



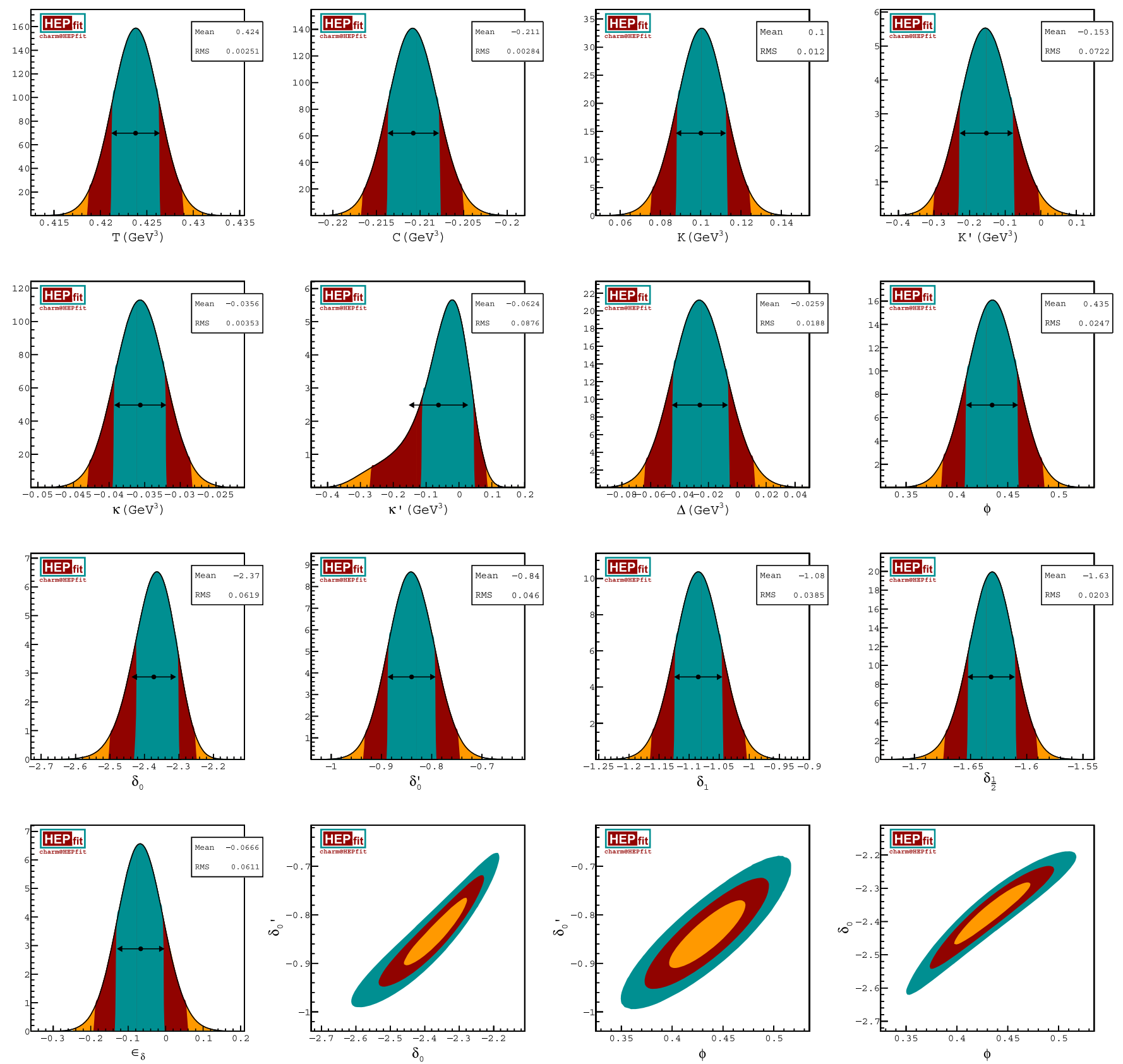

FIG. 6. The marginalized posterior distributions of the parameters from the fit as given in Table III. The green, red, and orange regions are the $68 \%, 95 \%$, and $99 \%$ probability regions respectively. The bottom three 2D marginalized plots show the correlations between the parameters $\delta_{0}, \delta_{0}^{\prime}$, and $\phi$, The orange, red, and green regions are the $68 \%, 95 \%$, and $99 \%$ probability regions, respectively.

[1] N. Cabibbo, Leptonic Decays in the unitary symmetry scheme, in Proceedings of the International Conference on Fundamental Aspects of Weak Interactions, BNL, Upton, NY, USA, 1963 (Brookhaven National Laboratory, Upton, NY, 1964), pp. 299-302.
[2] N. Cabibbo, Unitary Symmetry and Leptonic Decays, Phys. Rev. Lett. 10, 531 (1963).

[3] M. Kobayashi and T. Maskawa, $C P$ violation in the renormalizable theory of weak interaction, Prog. Theor. Phys. 49, 652 (1973). 
[4] J. R. Batley et al. (NA48 Collaboration), A precision measurement of direct $C P$ violation in the decay of neutral kaons into two pions, Phys. Lett. B 544, 97 (2002).

[5] A. Alavi-Harati et al. (KTeV Collaboration), Measurements of direct $C P$ violation, $C P T$ symmetry, and other parameters in the neutral kaon system, Phys. Rev. D 67, 012005 (2003); Erratum, Phys. Rev. D 70, 079904(E) (2004).

[6] E. Abouzaid et al. (KTeV Collaboration), Precise measurements of direct $C P$ violation, $C P T$ symmetry, and other parameters in the neutral Kaon system, Phys. Rev. D 83, 092001 (2011).

[7] L. Wolfenstein, Violation of $C P$ Invariance and the Possibility of Very Weak Interactions, Phys. Rev. Lett. 13, 562 (1964).

[8] Z. Bai et al. (RBC and UKQCD Collaborations), Standard Model Prediction for Direct $C P$ Violation in $K \rightarrow \pi \pi$ Decay, Phys. Rev. Lett. 115, 212001 (2015).

[9] K. F. Chen et al. (Belle Collaboration), Observation of Time-Dependent $C P$ Violation in $B^{0} \rightarrow \eta^{\prime} K^{0}$ Decays and Improved Measurements of $C P$ Asymmetries in $B^{0} \rightarrow \phi K^{0}, K_{S}^{0} K_{S}^{0} K_{S}^{0}$, and $B^{0} \rightarrow J / \psi K^{0}$ Decays, Phys. Rev. Lett. 98, 031802 (2007).

[10] B. Aubert et al. (BABAR Collaboration), Improved Measurement of $C P$ Violation in Neutral $B$ Decays to $c \bar{c}$ s, Phys. Rev. Lett. 99, 171803 (2007).

[11] B. Aubert et al. (BABAR Collaboration), Measurement of time-dependent $C P$ asymmetry in $B^{0} \rightarrow c \bar{c} K^{(*) 0}$ decays, Phys. Rev. D 79, 072009 (2009).

[12] I. Adachi et al., Precise Measurement of the $C P$ Violation Parameter $\sin 2 \phi_{1}$ in $B^{0} \rightarrow(c \bar{c}) K^{0}$ Decays, Phys. Rev. Lett. 108, 171802 (2012).

[13] M. Bander, D. Silverman, and A. Soni, CP Noninvariance in the Decays of Heavy Charged Quark Systems, Phys. Rev. Lett. 43, 242 (1979).

[14] A. B. Carter and A. I. Sanda, $C P$ violation in $B$ meson decays, Phys. Rev. D 23, 1567 (1981).

[15] I. I. Y. Bigi and A. I. Sanda, Notes on the observability of $C P$ violations in $B$ decays, Nucl. Phys. B193, 85 (1981).

[16] R. Aaij et al. (LHCb Collaboration), Precision Measurement of $C P$ Violation in $B_{s}^{0} \rightarrow J / \psi K^{+} K^{-}$Decays, Phys. Rev. Lett. 114, 041801 (2015).

[17] E. M. Aitala et al. (E791 Collaboration), Search for $D^{0}-\bar{D}^{0}$ Mixing in Semileptonic Decay Modes, Phys. Rev. Lett. 77, 2384 (1996).

[18] C. Cawlfield et al. (CLEO Collaboration), Limits on neutral $D$ mixing in semileptonic decays, Phys. Rev. D 71, 077101 (2005).

[19] B. Aubert et al. (BABAR Collaboration), Search for $D^{0}-\bar{D}^{0}$ mixing using doubly flavor tagged semileptonic decay modes, Phys. Rev. D 76, 014018 (2007).

[20] U. Bitenc et al. (Belle Collaboration), Improved search for $D^{0}-\bar{D}^{0}$ mixing using semileptonic decays at Belle, Phys. Rev. D 77, 112003 (2008).

[21] E. M. Aitala et al. (E791 Collaboration), A search for $D^{0}-\bar{D}^{0}$ mixing and doubly Cabibbo suppressed decays of the $D^{0}$ in hadronic final states, Phys. Rev. D 57, 13 (1998).

[22] R. Godang et al. (CLEO Collaboration), Search for $D^{0}-\bar{D}^{0}$ Mixing, Phys. Rev. Lett. 84, 5038 (2000).
[23] J. M. Link et al. (FOCUS Collaboration), Measurement of the doubly Cabibbo suppressed decay $D^{0} \rightarrow K^{+} p i^{-}$and a search for charm mixing, Phys. Lett. B 618, 23 (2005).

[24] L. M. Zhang et al. (Belle Collaboration), Improved Constraints on $D^{0}-\bar{D}^{0}$ Mixing in $D^{0} \rightarrow K^{+} \pi^{-}$Decays from the Belle Detecotr, Phys. Rev. Lett. 96, 151801 (2006).

[25] B. Aubert et al. (BABAR Collaboration), Evidence for $D^{0}-\bar{D}^{0}$ Mixing, Phys. Rev. Lett. 98, 211802 (2007).

[26] T. A. Aaltonen et al. (CDF Collaboration), Observation of $D^{0}-\bar{D}^{0}$ Mixing Using the CDF II Detector, Phys. Rev. Lett. 111, 231802 (2013).

[27] B. R. Ko et al. (Belle Collaboration), Observation of $D^{0}-\bar{D}^{0}$ Mixing in $e^{+} e^{-}$Collisions, Phys. Rev. Lett. 112, 111801 (2014); Publisher's Note: Observation of $D^{0}-\bar{D}^{0}$ Mixing in $e^{+} e^{-}$Collisions, Phys. Rev. Lett. 112, 139903 (2014).

[28] B. Aubert et al. (BABAR Collaboration), Measurement of $D^{0}-\bar{D}^{0}$ Mixing from a Time-Dependent Amplitude Analysis of $D^{0} \rightarrow K^{+} \pi^{-} \pi^{0}$ Decays, Phys. Rev. Lett. 103, 211801 (2009).

[29] E. M. Aitala et al. (E791 Collaboration), Measurements of Lifetimes and a Limit on the Lifetime Difference in the Neutral D Meson System, Phys. Rev. Lett. 83, 32 (1999).

[30] J. M. Link et al. (FOCUS Collaboration), A measurement of lifetime differences in the neutral $D$ meson system, Phys. Lett. B 485, 62 (2000).

[31] S. E. Csorna et al. (CLEO Collaboration), Lifetime differences, direct $C P$ violation and partial widths in $D^{0}$ meson decays to $K^{+} K^{-}$and $p i^{+} p i^{-}$, Phys. Rev. D 65 , 092001 (2002).

[32] K. Abe et al. (Belle Collaboration), Measurement of Lifetime Difference in $D^{0}$ Meson Decays, Phys. Rev. Lett. 88, 162001 (2002).

[33] J. P. Lees et al. (BABAR Collaboration), Measurement of $D^{0}-\bar{D}^{0}$ mixing and $C P$ violation in two-body $D^{0}$ decays, Phys. Rev. D 87, 012004 (2013).

[34] M. Staric (Belle Collaboration), New Belle results on $D^{0}-\bar{D}^{0}$ mixing, arXiv:1212.3478.

[35] T. A. Aaltonen et al. (CDF Collaboration), Measurement of indirect CP-violating asymmetries in $D^{0} \rightarrow K^{+} K^{-}$and $D^{0} \rightarrow \pi^{+} \pi^{-}$decays at CDF, Phys. Rev. D 90, 111103 (2014).

[36] B. R. Ko (Belle Collaboration), $C P$ violation and mixing in the charm sector at Belle, and current HFAG averages, arXiv:1212.5320.

[37] P. del Amo Sanchez et al. (BABAR Collaboration), Measurement of $D^{0}-\bar{D}^{0}$ Mixing Parameters Using $D^{0} \rightarrow K_{S}^{0} \pi^{+} \pi^{-}$and $D^{0} \rightarrow K_{S}^{0} K^{+} K^{-}$Decays, Phys. Rev. Lett. 105, 081803 (2010).

[38] T. Peng et al. (Belle Collaboration), Measurement of $D^{0}-\bar{D}^{0}$ mixing and search for indirect $C P$ violation using $D^{0} \rightarrow K_{S}^{0} \pi^{+} \pi^{-}$decays, Phys. Rev. D 89, 091103 (2014).

[39] J. P. Lees et al. (BABAR Collaboration), Measurement of the neutral $D$ meson mixing parameters in a timedependent amplitude analysis of the $D^{0} \rightarrow \pi^{+} \pi^{-} \pi^{0}$ decay, Phys. Rev. D 93, 112014 (2016).

[40] D. M. Asner et al. (CLEO Collaboration), Updated measurement of the strong phase in $D^{0} \rightarrow K^{+} \pi^{-}$decay using 
quantum correlations in $e^{+} e^{-} \rightarrow D^{0} \bar{D}^{0}$ at CLEO, Phys. Rev. D 86, 112001 (2012).

[41] B. Aubert et al. (BABAR Collaboration), Search for $C P$ Violation in the Decays $D^{0} \rightarrow K^{-} K^{+}$and $D^{0} \rightarrow \pi^{-} \pi^{+}$, Phys. Rev. Lett. 100, 061803 (2008).

[42] A. Di Canto, $C P$ violation in charm decays at CDF, Nuovo Cimento C 36, 26 (2013).

[43] R. Aaij et al. (LHCb Collaboration), Measurement of indirect $C P$ asymmetries in $D^{0} \rightarrow K^{-} K^{+}$and $D^{0} \rightarrow \pi^{-} \pi^{+}$ decays using semileptonic $B$ decays, J. High Energy Phys. 04 (2015) 043.

[44] R. Aaij et al. (LHCb Collaboration), Measurement of $C P$ asymmetry in $D^{0} \rightarrow K^{-} K^{+}$and $D^{0} \rightarrow \pi^{-} \pi^{+}$decays, J. High Energy Phys. 07 (2014) 041.

[45] R. Aaij et al. (LHCb Collaboration), Measurement of the Difference of Time-Integrated $C P$ Asymmetries in $D^{0} \rightarrow$ $K^{-} K^{+}$and $D^{0} \rightarrow \pi^{-} \pi^{+}$Decays, Phys. Rev. Lett. 116, 191601 (2016).

[46] R. Aaij et al. (LHCb Collaboration), Measurements of charm mixing and $C P$ violation using $D^{0} \rightarrow K^{ \pm} \pi^{\mp}$ decays, Phys. Rev. D 95, 052004 (2017).

[47] R. Aaij et al. (LHCb Collaboration), First Observation of $D^{0}-\bar{D}^{0}$ Oscillations in $D^{0} \rightarrow K^{+} \pi^{-} \pi^{+} \pi^{-}$Decays and Measurement of the Associated Coherence Parameters, Phys. Rev. Lett. 116, 241801 (2016).

[48] R. Aaij et al. (LHCb Collaboration), Measurement of mixing and $C P$ violation parameters in two-body charm decays, J. High Energy Phys. 04 (2012) 129.

[49] LHCb Collaboration, $C P$-violating asymmetries from the decay-time distribution of prompt $D^{0} \rightarrow K^{+} K^{-}$and $D^{0} \rightarrow$ $\pi^{+} \pi^{-}$decays in the full LHCb Run 1 data sample. Measurement using yield asymmetries in bins of decay time, LHCb Reports No. LHCb-CONF-2016-009 and No. CERN-LHCb-CONF-2016-009， 2016, http://cds .cern.ch/record/2220079.

[50] R. Aaij et al. (LHCb Collaboration), Model-independent measurement of mixing parameters in $D^{0} \rightarrow K_{S}^{0} \pi^{+} \pi^{-}$ decays, J. High Energy Phys. 04 (2016) 033.

[51] T. Aaltonen et al. (CDF Collaboration), Measurement of the Difference of $\mathrm{CP}$-Violating Asymmetries in $D^{0} \rightarrow$ $K^{+} K^{-}$and $D^{0} \rightarrow \pi^{+} \pi^{-}$Decays at CDF, Phys. Rev. Lett. 109, 111801 (2012).

[52] R. Aaij et al. (LHCb Collaboration), Evidence for $C P$ Violation in Time-Integrated $D^{0} \rightarrow h^{-} h^{+}$Decay Rates, Phys. Rev. Lett. 108, 111602 (2012).

[53] B. R. Ko (Belle Collaboration), Direct $C P$ violation in charm at Belle, Proc. Sci., ICHEP2012 (2013) 353 [arXiv:1212.1975].

[54] R. Aaij et al. (LHCb Collaboration), Search for direct $C P$ violation in $D 0 \rightarrow h^{-} h^{+}$modes using semileptonic $B$ decays, Phys. Lett. B 723, 33 (2013).

[55] I. I. Bigi, A. Paul, and S. Recksiegel, Conclusions from CDF results on $C P$ violation in $D^{0} \rightarrow \pi^{+} \pi^{-}, K^{+} K^{-}$and future Tasks, J. High Energy Phys. 06 (2011) 089.

[56] Y. Grossman, A. L. Kagan, and Y. Nir, New physics and $C P$ violation in singly Cabibbo suppressed $D$ decays, Phys. Rev. D 75, 036008 (2007).

[57] G. Isidori, J. F. Kamenik, Z. Ligeti, and G. Perez, Implications of the $\mathrm{LHCb}$ evidence for charm $C P$ violation, Phys. Lett. B 711, 46 (2012).
[58] K. Wang and G. Zhu, Can up FCNC solve the $\Delta A_{\mathrm{CP}}$ puzzle?, Phys. Lett. B 709, 362 (2012).

[59] G. Hiller, Y. Hochberg, and Y. Nir, Supersymmetric $\Delta A_{\mathrm{CP}}$, Phys. Rev. D 85, 116008 (2012).

[60] G. F. Giudice, G. Isidori, and P. Paradisi, Direct $C P$ violation in charm and flavor mixing beyond the SM, J. High Energy Phys. 04 (2012) 060.

[61] W. Altmannshofer, R. Primulando, C.-T. Yu, and F. Yu, New physics models of direct $C P$ violation in charm decays, J. High Energy Phys. 04 (2012) 049.

[62] C.-H. Chen, C.-Q. Geng, and W. Wang, CP violation in $D^{0} \rightarrow\left(K^{-} K^{+}, \pi^{-} \pi^{+}\right)$from diquarks, Phys. Rev. D 85, 077702 (2012).

[63] O. Gedalia, J. F. Kamenik, Z. Ligeti, and G. Perez, On the universality of $C P$ violation in $\Delta F=1$ processes, Phys. Lett. B 714, 55 (2012).

[64] T. Mannel and N. Uraltsev, Charm $C P$ violation and the electric dipole moments from the charm scale, J. High Energy Phys. 03 (2013) 064.

[65] B. Keren-Zur, P. Lodone, M. Nardecchia, D. Pappadopulo, R. Rattazzi, and L. Vecchi, On partial compositeness and the CP asymmetry in charm decays, Nucl. Phys. B867, 394 (2013).

[66] R. Barbieri, D. Buttazzo, F. Sala, and D. M. Straub, Less minimal flavour violation, J. High Energy Phys. 10 (2012) 040 .

[67] A. D. Dolgov, S. I. Godunov, A. N. Rozanov, and M. I. Vysotsky, Charmed penguin versus BAU, JETP Lett. 96, 290 (2012).

[68] C. Delaunay, J. F. Kamenik, G. Perez, and L. Randall, Charming $C P$ violation and dipole operators from RS flavor anarchy, J. High Energy Phys. 01 (2013) 027.

[69] M. Golden and B. Grinstein, Enhanced CP violations in hadronic charm decays, Phys. Lett. B 222, 501 (1989).

[70] D. Pirtskhalava and P. Uttayarat, $C P$ violation and flavor $S U(3)$ breaking in $D$-meson decays, Phys. Lett. B 712, 81 (2012).

[71] B. Bhattacharya, M. Gronau, and J. L. Rosner, $C P$ asymmetries in singly-Cabibbo-suppressed $D$ decays to two pseudoscalar mesons, Phys. Rev. D 85, 054014 (2012).

[72] T. Feldmann, S. Nandi, and A. Soni, Repercussions of flavour symmetry breaking on $C P$ violation in $D$-meson decays, J. High Energy Phys. 06 (2012) 007.

[73] J. Brod, A. L. Kagan, and J. Zupan, Size of direct $C P$ violation in singly Cabibbo-suppressed $D$ decays, Phys. Rev. D 86, 014023 (2012).

[74] J. Brod, Y. Grossman, A. L. Kagan, and J. Zupan, A consistent picture for large penguins in $D \rightarrow \pi+\pi-$, $K^{+} K^{-}$, J. High Energy Phys. 10 (2012) 161.

[75] H.-Y. Cheng and C.-W. Chiang, $S U(3)$ symmetry breaking and $C P$ violation in $D \rightarrow P P$ decays, Phys. Rev. D 86, 014014 (2012).

[76] G. Hiller, M. Jung, and S. Schacht, $S U(3)$-flavor anatomy of nonleptonic charm decays, Phys. Rev. D 87, 014024 (2013).

[77] A. Le Yaouanc, L. Oliver, and J. C. Raynal, CP violation beyond the standard model and final state interaction phases in D mesons, Phys. Lett. B 292, 353 (1992). 
[78] E. Franco, S. Mishima, and L. Silvestrini, The standard model confronts $C P$ violation in $D^{0} \rightarrow \pi^{+} \pi^{-}$and $D^{0} \rightarrow K^{+} K^{-}$, J. High Energy Phys. 05 (2012) 140.

[79] F. Buccella, M. Lusignoli, G. Miele, A. Pugliese, and P. Santorelli, Nonleptonic weak decays of charmed mesons, Phys. Rev. D 51, 3478 (1995).

[80] F. Buccella, M. Lusignoli, and A. Pugliese, Charm nonleptonic decays and final state interactions, Phys. Lett. B 379, 249 (1996).

[81] S. Müller, U. Nierste, and S. Schacht, Topological amplitudes in $D$ decays to two pseudoscalars: A global analysis with linear $S U(3)_{F}$ breaking, Phys. Rev. D 92, 014004 (2015).

[82] S. Müller, U. Nierste, and S. Schacht, Sum Rules of Charm $C P$ Asymmetries Beyond the $\mathrm{SU}(3)_{F}$ Limit, Phys. Rev. Lett. 115, 251802 (2015).

[83] U. Nierste and S. Schacht, $C P$ Violation in $D^{0} \rightarrow K_{S} K_{S}$, Phys. Rev. D 92, 054036 (2015).

[84] M. Ciuchini, E. Franco, G. Martinelli, and L. Silvestrini, Charming penguins in $B$ decays, Nucl. Phys. B501, 271 (1997).

[85] A. J. Buras and L. Silvestrini, Nonleptonic two-body $B$ decays beyond factorization, Nucl. Phys. B569, 3 (2000).

[86] P. Zenczykowski, Nonleptonic charmed meson decays: Quark diagrams and final-state interactions, Acta Phys. Pol. B 28, 1605 (1997).

[87] H.-Y. Cheng, Weak annihilation and the effective parameters a(1) and a(2) in nonleptonic D decays, Eur. Phys. J. C 26, 551 (2003).

[88] For recent alternate approaches, see Refs. [89,90].

[89] H.-n. Li, C.-D. Lu, and F.-S. Yu, Branching ratios and direct $C P$ asymmetries in $D \rightarrow P P$ decays, Phys. Rev. D 86, 036012 (2012).

[90] A. Biswas, N. Sinha, and G. Abbas, Nonleptonic decays of charmed mesons into two pseudoscalars, Phys. Rev. D 92 , 014032 (2015).

[91] F. Buccella, M. Lusignoli, A. Pugliese, and P. Santorelli, $C P$ violation in $D$ meson decays: Would it be a sign of new physics?, Phys. Rev. D 88, 074011 (2013).

[92] B. Bhattacharya and J. L. Rosner, Charmed meson decays to two pseudoscalars, Phys. Rev. D 81, 014026 (2010).

[93] P. Ball, J. M. Frere, and M. Tytgat, Phenomenological evidence for the gluon content of $\eta$ and $\eta^{\prime}$, Phys. Lett. B 365, 367 (1996).

[94] C. Di Donato, G. Ricciardi, and I. Bigi, $\eta-\eta^{\prime}$ mixing: From electromagnetic transitions to weak decays of charm and beauty hadrons, Phys. Rev. D 85, 013016 (2012).

[95] T. Feldmann, Quark structure of pseudoscalar mesons, Int. J. Mod. Phys. A 15, 159 (2000).

[96] The approximate selection rule that leads to the suppression of the simultaneous creation of $d \bar{d}$ and $s \bar{s}$ pairs in our framework is analogous to a Zweig suppression (or OZI rule) [97-99]. We refrain from calling it the Zweig suppression since the process $\phi \rightarrow \rho \pi$, forbidden by the Zweig rule, might occur by allowing the conversion of the initial $s \bar{s}$ into a pair of light quarks accompanied by the creation of another pair of light quarks. To exclude the formation of a $K^{0} \bar{K}^{0}$ pair through the $\Delta U=0$ decay amplitude we do not allow the conversion of the $u \bar{u}$ pair into a $d \bar{d}$ or $s \bar{s}$ pair and assume that the constituents of the final mesons are just the three quarks produced in the $c$ decay and the spectator $\bar{q}$. This has a consequence also for the $\Delta U=1$ part related by $\mathrm{SU}(3)_{F}$ to the $\Delta U=0$. Our selection rule is better motivated, since asymptotic freedom implies that the strong coupling constant is a decreasing function of the scale.

[97] S. Okubo, $\Phi$-meson and unitary symmetry model, Phys. Lett. 5, 165 (1963).

[98] G. Zweig, An $\mathrm{SU}_{3}$ model for strong interaction symmetry and its breaking, Version 2, CERN Report No. CERN-TH412, 1964, p. 80, http://cds.cern.ch/record/570209; version 1 is CERN preprint 8182/TH.401, 1964.

[99] J. Iizuka, Systematics and phenomenology of meson family, Prog. Theor. Phys. Suppl. 37, 21 (1966).

[100] D. Atwood and A. Soni, Searching for the origin of $C P$ violation in Cabibbo suppressed D-meson decays, Prog. Theor. Exp. Phys. 2013, 093 B05 (2013).

[101] H.-Y. Cheng and C.-W. Chiang, Two-body hadronic charmed meson decays, Phys. Rev. D 81, 074021 (2010).

[102] Y. Amhis et al., Averages of $b$-hadron, $c$-hadron, and $\tau$-lepton properties as of summer 2016, Eur. Phys. J. C 77, 895 (2017).

[103] R. Aaij et al. (LHCb Collaboration), Measurements of Indirect $C P$ Asymmetries in $D^{0} \rightarrow K^{-} K^{+}$and $D^{0} \rightarrow$ $\pi^{-} \pi^{+}$Decays, Phys. Rev. Lett. 112, 041801 (2014).

[104] R. Aaij et al. (LHCb Collaboration), Observation of $C P$ violation in charm decays, arXiv:1903.08726.

[105] G. Bonvicini et al. (CLEO Collaboration), Search for $C P$ violation in $D^{0} \rightarrow K_{S}^{0} \pi^{0}$ and $D^{0} \rightarrow \pi^{0} \pi^{0}$ and $D^{0} \rightarrow K_{S}^{0} K_{S}^{0}$ decays, Phys. Rev. D 63, 071101 (2001).

[106] N. K. Nisar et al. (Belle Collaboration), Search for $C P$ Violation in $D^{0} \rightarrow \pi^{0} \pi^{0}$ Decays, Phys. Rev. Lett. 112, 211601 (2014).

[107] J. M. Link et al. (FOCUS Collaboration), Search for $C P$ Violation in the Decays $D^{+} \rightarrow K_{S} \pi^{+}$and $D^{+} \rightarrow K_{S} K^{+}$, Phys. Rev. Lett. 88, 041602 (2002); Erratum, Phys. Rev. Lett. 88, 159903(E) (2002).

[108] H. Mendez et al. (CLEO Collaboration), Measurements of $D$ meson decays to two pseudoscalar mesons, Phys. Rev. D 81, 052013 (2010).

[109] B. R. Ko et al. (Belle Collaboration), Search for $C P$ Violation in the Decay $D^{+} \rightarrow K_{S}^{0} K^{+}$, J. High Energy Phys. 02 (2013) 098.

[110] J. P. Lees et al. (BABAR Collaboration), Search for $C P$ violation in the decays $D^{ \pm} \rightarrow K_{S}^{0} K^{ \pm}, D_{s}^{ \pm} \rightarrow K_{S}^{0} K^{ \pm}$, and $D_{s}^{ \pm} \rightarrow K_{S}^{0} \pi^{ \pm}$, Phys. Rev. D 87, 052012 (2013).

[111] R. Aaij et al. (LHCb Collaboration), Measurement of the time-integrated $C P$ asymmetry in $D^{0} \rightarrow K_{S}^{0} K_{S}^{0}$ decays, J. High Energy Phys. 10 (2015) 055.

[112] A. Abdesselam et al., Measurement of $C P$ asymmetry in the $D^{0} \rightarrow K_{S}^{0} K_{S}^{0}$ decay at Belle, arXiv:1609.06393.

[113] F. Buccella, M. Lusignoli, G. Mangano, G. Miele, A. Pugliese, and P. Santorelli, CP violating asymmetries in charged $D$ meson decays, Phys. Lett. B 302, 319 (1993).

[114] Y. Grossman, A. L. Kagan, and J. Zupan, Testing for new physics in singly Cabibbo suppressed $D$ decays, Phys. Rev. D 85, 114036 (2012). 
[115] V. Babu et al. (for the Belle), Search for $C P$ violation in the $D^{+} \rightarrow \pi^{+} \pi^{0}$ decay at Belle, Phys. Rev. D 97, 011101 (2018).

[116] M. Ablikim et al. (BESIII Collaboration), Measurements of the absolute branching fractions and $C P$ asymmetries for $D^{+} \rightarrow K_{S, L}^{0} K^{+}\left(\pi^{0}\right)$, Phys. Rev. D 99, 032002 (2019).

[117] HEPFIT: A tool to combine indirect and direct constraints on High Energy Physics, http://hepfit.roma1.infn.it/.

[118] M. Ablikim et al. (BESIII Collaboration), Measurements of absolute branching fractions for $D$ mesons decays into two pseudoscalar mesons, Phys. Rev. D 97, 072004 (2018).

[119] C. Patrignani et al. (Particle Data Group), Review of particle physics, Chin. Phys. C 40, 100001 (2016).

[120] A. Zupanc et al. (Belle Collaboration), Measurements of branching fractions of leptonic and hadronic $D_{s}^{+}$meson decays and extraction of the $D_{s}^{+}$meson decay constant, J. High Energy Phys. 09 (2013) 139.

[121] S. Okubo, Note on unitary symmetry in strong interactions, Prog. Theor. Phys. 27, 949 (1962).

[122] Y. Ne'eman, Derivation of strong interactions from a gauge invariance, Nucl. Phys. 26, 222 (1961).

[123] S. Okubo, Note on unitary symmetry in strong interaction. II Excited states of baryons, Prog. Theor. Phys. 28, 24 (1962).

[124] M. Gell-Mann, Symmetries of baryons and mesons, Phys. Rev. 125, 1067 (1962).

[125] D. Wang, F.-S. Yu, P.-F. Guo, and H.-Y. Jiang, $K_{S}^{0}-K_{L}^{0}$ asymmetries in $D$-meson decays, Phys. Rev. D 95, 073007 (2017).

[126] The average corresponds to the one presented by HFLAV post-Moriond 2019.

[127] The predicted value has the opposite sign for the positive solutions of the strong phases.

[128] L.-L. Chau and H.-Y. Cheng, SU(3) breaking effects in charmed meson decays, Phys. Lett. B 333, 514 (1994).

[129] T. E. Browder and S. Pakvasa, Experimental implications of large $C P$ violation and final state interactions in the search for $D^{0}-\bar{D}^{0}$ mixing, Phys. Lett. B 383, 475 (1996).

[130] A. F. Falk, Y. Nir, and A. A. Petrov, Strong phases and $D^{0}-\bar{D}^{0}$ mixing parameters, J. High Energy Phys. 12 (1999) 019.
[131] D.-N. Gao, Strong phases, asymmetries, and SU(3) symmetry breaking in $D \rightarrow K \pi$ decays, Phys. Lett. B 645, 59 (2007).

[132] R. L. Kingsley, S. B. Treiman, F. Wilczek, and A. Zee, Weak decays of charmed hadrons, Phys. Rev. D 11, 1919 (1975).

[133] M. B. Voloshin, V. I. Zakharov, and L. B. Okun, Two particle nonleptonic decays of $D$ and $F$ mesons, and structure of weak interactions, Pis'ma Zh. Eksp. Teor. Fiz. 21, 403 (1975) [JETP Lett. 21, 183 (1975)].

[134] L. Wolfenstein, $C P$ Violation in $D^{0}-\bar{D}^{0}$ Mixing, Phys. Rev. Lett. 75, 2460 (1995).

[135] I. I. Y. Bigi and H. Yamamoto, Interference between Cabibbo allowed and doubly forbidden transitions in $D \rightarrow K_{S}, K_{L}+\pi$ 's decays, Phys. Lett. B 349, 363 (1995).

[136] Q. He et al. (CLEO Collaboration), Comparison of $D \rightarrow K_{S}^{0} \pi$ and $D \rightarrow K_{L}^{0} \pi$ Decay Rates, Phys. Rev. Lett. 100, 091801 (2008).

[137] M. Gronau, $S U(3)$ in $D$ decays: From $30 \%$ symmetry breaking to $10^{-4}$ precision, Phys. Rev. D 91, 076007 (2015).

[138] The posterior distributions of all three observables are nonGaussian and hence, the error bars have been interpreted as the rms of the distributions.

[139] B. Bhattacharya and J. L. Rosner, Flavor symmetry and decays of charmed mesons to pairs of light pseudoscalars, Phys. Rev. D 77, 114020 (2008).

[140] D.-N. Gao, Asymmetries from the interference between Cabibbo-favored and doubly-Cabibbo-suppressed $D$ meson decays, Phys. Rev. D 91, 014019 (2015).

[141] The predicted value is the same for both the solutions of the strong phases, negative and positive.

[142] A. J. Schwartz, Charm physics prospects at Belle II, Proc. Sci., CHARM2016 (2017) 042 [arXiv:1701.07159].

[143] LHCb Collaboration, Impact of the LHCb upgrade detector design choices on physics and trigger performance, CERN Technical Reports No. LHCb-PUB-2014-040, No. CERN-LHCb-PUB-2014-040, and No. LHCb-INT2013-024, 2014, https://cds.cern.ch/record/1748643.

[144] M. Bona et al. (UTfit Collaboration), The unitarity triangle fit in the standard model and hadronic parameters from lattice QCD: A reappraisal after the measurements of $\Delta m_{s}$ and $\operatorname{BR}\left(B \rightarrow \tau \nu_{\tau}\right)$, J. High Energy Phys. 10 (2006) 081 . 